Article

\title{
A Group Replacement Decision Support System Based on Internet of Things
}

\author{
Gia-Shie Liu
}

Department of Management, Lunghwa University of Science and Technology, Taoyuan 33306, Taiwan; liugtw@yahoo.com.tw or liug@gm.lhu.edu.tw

Received: 11 August 2019; Accepted: 26 August 2019; Published: 2 September 2019

check for updates

\begin{abstract}
This paper combines computer-based monitoring technologies and Internet of things (IoT) technology to develop IoT condition-based group replacement decision support system for a production/service system with numerous parallel independent operating servers. This proposed IoT conditioned-based group replacement decision support system first develops the discounted cost model for a service/production system with numerous independent working servers. The original discounted cost model is further revised into an equivalent model to stimulate the proof procedure by applying the uniformization approach. Several significant theoretical properties are proved and many numerical examples are conducted for two kinds of group replacement policies, respectively. The first class of group replacement policy is developed and proved theoretically that there is a threshold of amount of customers existed to activate the group replacement depending on various amount of operating servers; numerical examples conducted in this study can also illustrate the above theoretical outcomes already derived for the first class of group replacement policy. Besides, for the second class of group replacement policy, the results of numerical examples definitely demonstrate that there is a threshold of the amount of operating servers needed to start the group replacement according to distinct amount of customers in the system. This proposed IoT condition-based group replacement decision support system derives the structure and detailed procedure flow to actually conduct the group replacement operations for many practical service or production systems.
\end{abstract}

Keywords: group replacement; condition-based replacement; decision support system; unreliable Markovian service systems; Internet of things

\section{Introduction}

To keep production/service system manipulating normally to run production line successfully or offer suitable services for customers is very significant in such customer-oriented business world. On the contrary, the delay of production flow or the loss of customers would have occurred and caused a lot of cost. In addition to built-in reliability design, a desirable maintenance policy also plays an important part in avoiding the breakdown of an operating system. Recently, there has been much research concerning maintenance and replacement problems. With regard to single-unit systems, different replacement policies including age-replacement [1-10], preventive replacement [11-14], failure limit replacement, and block replacement [5] are proposed, combined with minimal repair [5], unplanned replacement, warranty policy $[5,13,14]$, reliability criteria and other options depending on different conditions.

Age replacement implies that a system is replaced at a specified replacement age or at failure, whichever occurs first. Chien [3] establishes a completely renewable age-replacement policy combined with a proportional warranty policy. Park and Pham [5] deals with the optimal maintenance policy, block replacement policy and age replacement policy under renewable and non-renewable warranty policies. Lim et al. [4] presents age replacement policy depending on imperfect repair with random 
probability. The developed policy comprises the situation that such failure can be either perfectly repaired or minimally repaired with random probabilities. Concerning the on-line information, an adaptive age replacement policy is proposed for systems under changeable manipulating conditions using a cumulative exposure model [6]. Preventive replacement means that the care and service for maintaining equipment in approving operating condition by conducting systematic detection, and correction of failures either before they occur or before they become major defects. Dimitrakos and Kyriakidis [11] conducts the preventive maintenance installation as early as the degree of the system deterioration surpasses an extreme standard. Berthaut et al. [12] proposes a maintenance policy to establish the preventive maintenance schedule depending on the stock quantity. Wu et al. [13] considers a revenue-generating system to derive warranty contracts for a periodic preventive maintenance policy. Huang et al. [14] surveys an imperfect and periodic preventive maintenance strategy a product handled by base warranty and extended warranty region from the perspective of manufacturers.

Many of these replacement models for single-unit systems generally assume that all malfunctions will be immediately detected, repaired and replaced. However, it is not true in the real world. What frequently occurred is that the single-unit system must cease the production/service process for the malfunction recognition and further cause production loss or customer loss. A multi-unit system is the other option to prevent the pausing of related production/service process. According to our opinions, the replacement policies for multi-unit systems are usually derived from those for single-unit systems but more complex. Shafiee and Finkelstein [15] develops an age group replacement policy for a multi-server series system; the component is replaced by a new one when the degradation level reaches a given critical size, and the other components undergo a preventive maintenance action. A planned group preventive maintenance is conducted for the whole system at the proposed operational age. Babishin and Taghipour [16] considers a multicomponent system subject to hard and hidden soft failures and propose an optimal maintenance policy for each part and the optimal periodic examination for the system; this policy makes the maintenance decision according to the optimum age before replacement for the hard-type components and the optimum amount of minimal repairs until replacement for the soft-type components. Zhao et al. [7] presents a summary of review of age replacement models for a parallel multi-unit system with random number of units.

Several previous studies assume that the machines manufacture products with a consistent rate $[7,15,17-19]$. It means that related production loss cost incurred with a consistent rate when a failed machine is still kept un-repaired. Contrarily, this study considers that the production loss cost relies on the amount of products waiting for service in the system. Therefore, more accurate production loss cost can be acquired with this assumption. The group replacement problems focused in this study clearly belong to the field of unreliable queuing systems, which can be resolved by the matrix-geometric method [20,21]. Moreover, Liu [22] presents the group $m$-failure maintenance policies to deal with failure/repair process of servers and arrival/service process of customers at the same time; in total, three models are developed, two with positive repair time and one with instantaneous repair; the matrix geometric approach is employed to achieve the steady state distribution and further obtain the expected average cost for these three models. For the theoretical analysis, this paper demonstrates that there exists an optimal group replacement parameter $m^{*}$, which can acquire the minimal average cost for all three models. Moreover, several mathematical properties and sensitivity analyses are numerically demonstrated regarding different values of parameters. Consequently, the comparisons of these three proposed models in different situations are also discussed.

The above papers implementing the steady state analysis with matrix-geometric method can only get the expected average amount of customers, rather than let the replacement decision to dynamically depend on the amount of operating servers and customers in system [23]. Therefore, it is very important to be aware of the actual status of operating machines and the number of jobs waiting for process instantly. For achieving this goal, computer-based monitoring technologies and Internet of things technology can be applied in the maintenance model proposed in this paper. In recent years, research on condition-based maintenance has been speedily increasing because of 
the fast development of computer-based monitoring technologies. Condition-based maintenance is a maintenance strategy that gathers and evaluates real-time information, and suggests maintenance decisions based on the present status of the system. Olde Keizer et al. [18] conduct a literature review on condition-based maintenance policies focusing on multi-component systems subject to different dependences. Alaswad and Xiang [19] presents a review of condition-based maintenance literature with emphasis on various important aspects of optimization criteria, inspection frequency, maintenance degree, and solution methodology. Aizpurua et al. [24] proposes an online system maintenance method, which conducts an online predictive diagnosis algorithm to differentiate between critical and non-critical assets. A prognostics-updated method for estimating the system health is then conducted to yield well-informed, more precise, condition-based suggestions for the maintenance of critical components and for the group-based reactive repair of non-critical components. To take advantage of recent fast growing development of Internet of things, Li et al. [25] establishes predictive maintenance system which is depending on Internet of things technology to secure the mine safety monitoring and maintenance of equipment system.

Consequently, this paper tries to combine computer-based monitoring technologies and Internet of things (IoT) technology to develop IoT condition-based group replacement decision support system for an unreliable Markovian service system. Based on the structure and detailed procedure flow developed in this paper, the proposed IoT condition-based group replacement decision support system can be definitely conducted to solve the replacement problems of some designated service or production systems with several machines or servers manipulating in parallel.

The paper is organized as follows. Section 1 contains the background introduction and literature review. Section 2 describes the mathematical model formulation, and Section 3 depicts the related discounted cost formulation, and Section 4 illustrates the modified uniformization model. Furthermore, Section 5 demonstrates the related proofs of optimal policy and Section 6 contains numerical examples and results. Finally, Section 7 summarizes with the concluding remarks.

\section{Mathematical Model Formulation}

\subsection{Problem Description}

This study considers a production system with $M_{q n}$ servers connected in parallel in a single waiting line. Products enter in production line with a Poisson process of arrival rate $\sigma$, and the process time per product follows an exponential distribution with rate $\xi$. The servers fail with identically exponential distribution times and the breakdown rate of each server is $\beta$. In the replacement process, replacements are conducted by a crew of $\mathrm{j}$ repairmen. The replacement time for each server by using one repairman follows exponential distribution with rate $\gamma$. The overall replacement rate equals $\gamma_{m_{q n}}=\frac{j \gamma}{M_{q n}-m_{q n}}$, when $m_{q n}$ servers are normal and $M_{q n}-m_{q n}$ servers under replacement. The objective of this research is to build an IoT condition-based group replacement decision support system. The structure and the detailed procedure flow of our proposed decision support system (DSS) are shown in Figures 1 and 2, respectively. 


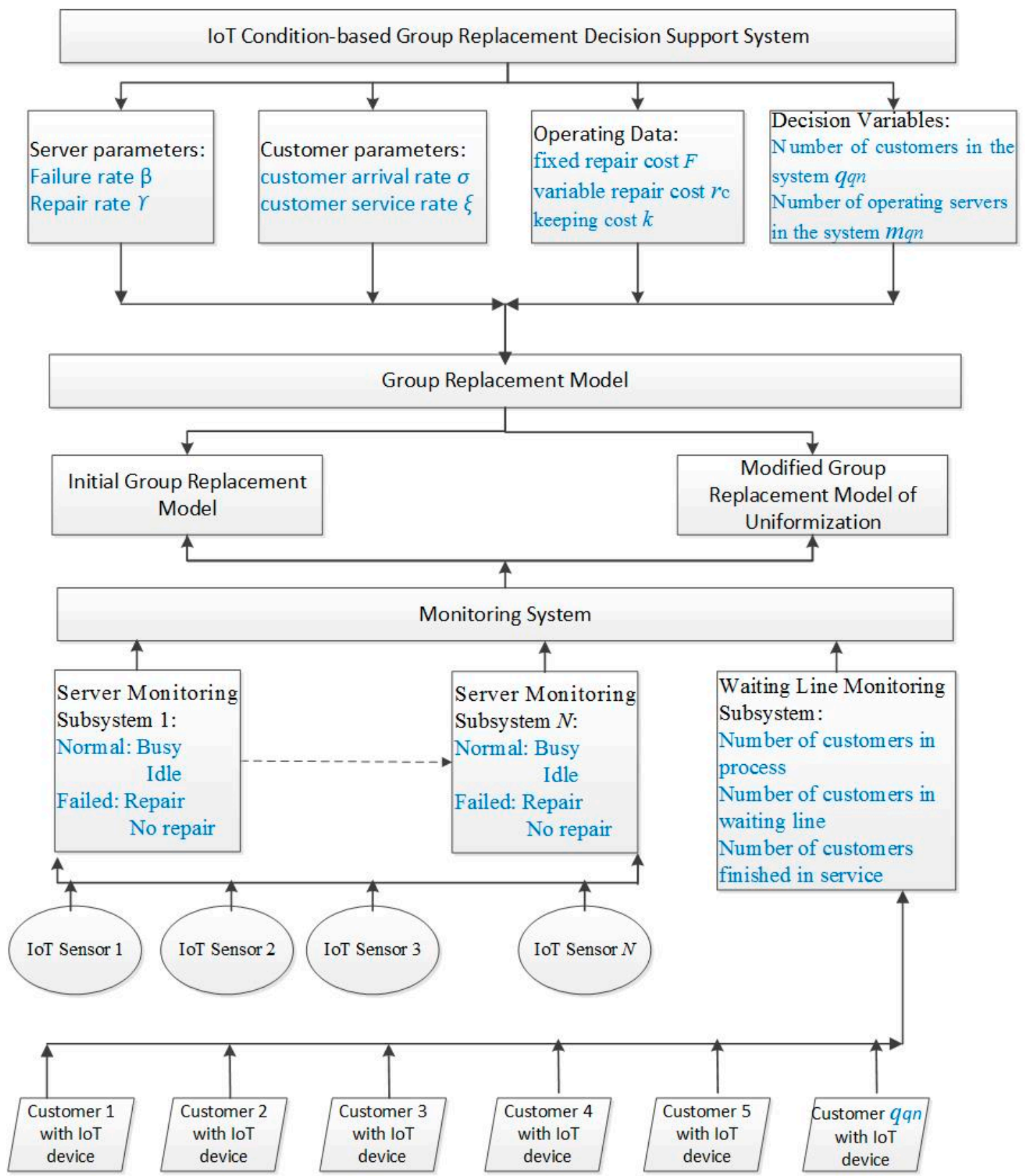

Figure 1. The structure of decision support system.

The main structure of our proposed decision support system shown in Figure 1 includes (1) Group Replacement Model Module: Initial Group Replacement Model and Modified Group Replacement Model of Uniformation; (2) Computer Monitoring System: Server Monitoring Subsystem and Waiting Line Monitoring Subsystem; (3) IoT Sensor Module: Servers with IoT devices and Customers with IoT devices. 


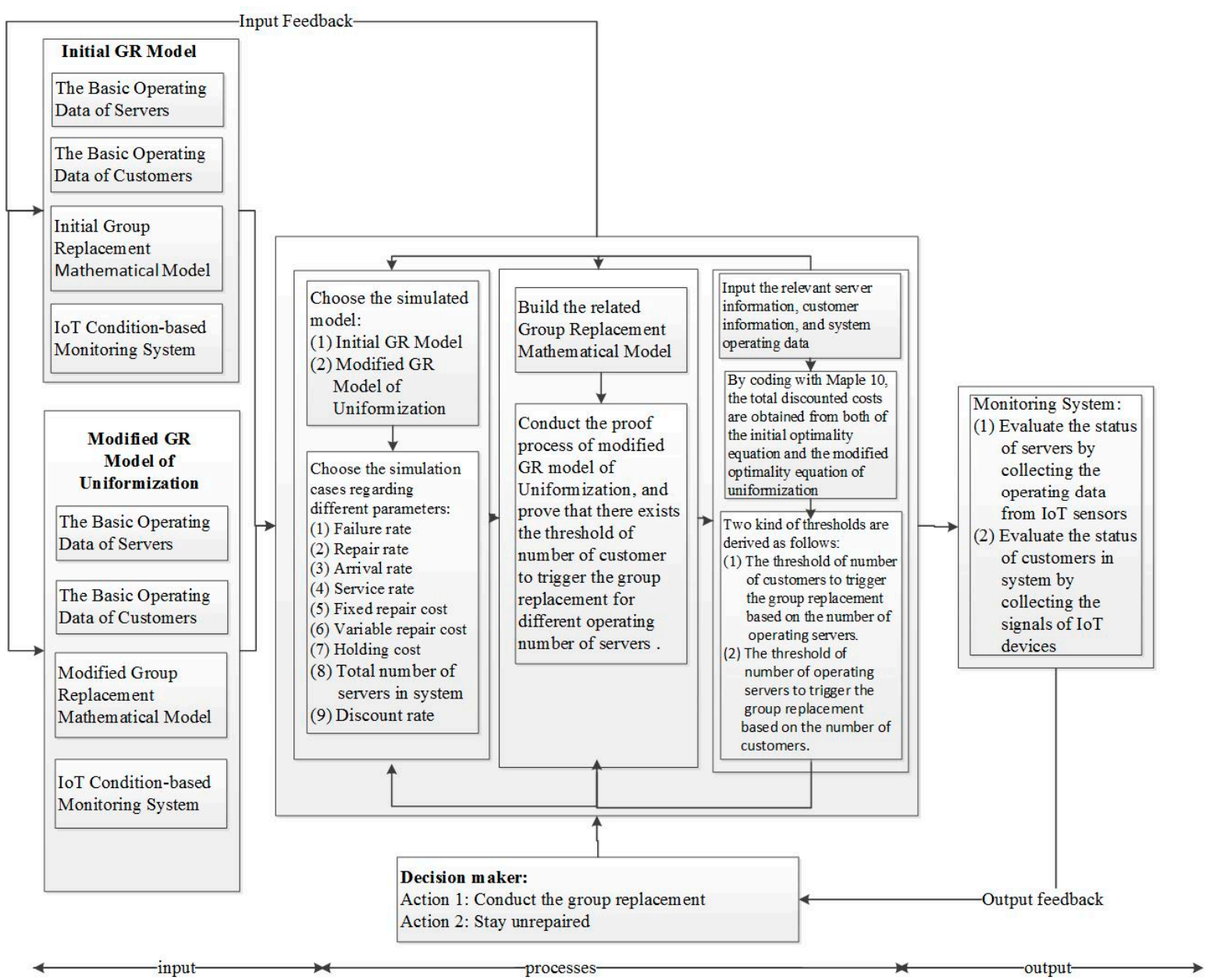

Figure 2. The procedure flow of decision support system.

The main procedure of our proposed decision support system is as follows:

Step 1: Choose the simulated group replacement model. Step 2: Choose the numerical cases regarding different parameters. Step 3: Build the related group replacement mathematical model. Step 4: Conduct the proof process of the Modified Group Replacement Model of Uniformation. Step 5: Several numerical cases are conducted to find the corresponding thresholds to trigger group replacements based on different operating servers or specific amount of customers in system for different values of parameters. Step 6: Computer monitoring system instantaneously evaluates the status of servers by collecting the precise operating data from desired IoT sensors, and the status of customers in system by collecting the signals of built-in IoT devices from these customers, and further decides whether the group replacement should be conducted based on the group replacement threshold.

\subsection{Group Replacement Mathematical Model Formulation}

The group replacement mathematical model of this proposed IoT condition-based decision support system can be modeled as a continuous-time Markov decision process. The parameters of the continuous-time Markov decision model can be specified as follows. The state space of the process is the set: $S=\{q, m\}$, where the state of the process is the state $\left(q_{q n}, m_{q n}\right), q_{q n} \geq 0$, and $0 \leq m_{q n} \leq M_{q n}$, where $q_{q n}$ indicates the amount of products in system, and $m_{q n}$ the amount of normal servers in system. The action set $\mathrm{A}\left(q_{q n}, m_{q n}\right)=\{1,2\}$, in which $a=1$ represents the production process is keeping on and $a=2$ indicates that the group replacement of breakdown servers is carried out. The transition probabilities for each action can be specified as follows. 
The mechanism of transition process for action $a=1$ (Proceed the production process) is $\left(q_{q n}, m_{q n}\right) \rightarrow\left(q_{q n}+1, m_{q n}\right)$ with arrival rate $\sigma ;\left(q_{q n}, m_{q n}\right) \rightarrow\left(q_{q n}, m_{q n}-1\right)$ with overall breakdown rate $m_{q n} \beta ;\left(q_{q n}, m_{q n}\right) \rightarrow\left(q_{q n}-1, m_{q n}\right)$ with overall process rate $\xi \min \left(q_{q n}, m_{q n}\right)$.

The mechanism of transition process for action $a=2$ (Cease the production process and conduct the group replacement $)$ is $\left(q_{q n}, m_{q n}\right) \rightarrow\left(q_{q n}+1, m_{q n}\right)$ with arrival rate $\sigma ;\left(q_{q n}, m_{q n}\right) \rightarrow\left(q_{q n}-1, m_{q n}\right)$ with overall process rate $\xi \min \left(q_{q n}, m_{q n}\right) ;\left(q_{q n}, m_{q n}\right) \rightarrow\left(q_{q n}, m_{q n}-1\right)$ with overall breakdown rate $m_{q n} \beta$; $\left(q_{q n}, m_{q n}\right) \rightarrow\left(q_{q n}, M_{q n}\right)$ the overall replacement rate $\gamma_{m_{q n}}$ equals $\frac{j \gamma}{M_{q n}-m_{q n}}$.

An equivalent transition mechanism can also be described as follows:

The transition mechanism for action $a=1$ means that the process will remain in $\left(q_{q n}, m_{q n}\right)$ for an exponential distribution time with overall rate $\sigma\left(q_{q n}, m_{q n}\right)=\sigma+m_{q n} \beta+\xi \min \left(q_{q n}, m_{q n}\right)$. After a transition takes place, it will head to one of $\left(q_{q n}+1, m_{q n}\right),\left(q_{q n}, m_{q n}-1\right),\left(q_{q n}-1, m_{q n}\right)$ with following specific probabilities:

$$
\begin{gathered}
P_{\left(q_{q n}, m_{q n}\right)\left(q_{q n}+1, m_{q n}\right)}(a=1)=\frac{\sigma}{\sigma\left(q_{q n}, m_{q n}\right)}, \\
P_{\left(q_{q n}, m_{q n}\right)\left(q_{q n}, m_{q n}-1\right)}(a=1)=\frac{m_{q n} \beta}{\sigma\left(q_{q n}, m_{q n}\right)}, \\
P_{\left(q_{q n}, m_{q n}\right)\left(q_{q n}-1, m_{q n}\right)}(a=1)=\frac{\xi \min \left(q_{q n}, m_{q n}\right)}{\sigma\left(q_{q n}, m_{q n}\right)} .
\end{gathered}
$$

The transition mechanism for action $a=2$ indicates that the process will remain in state $\left(q_{q n}, m_{q n}\right)$ for an exponentially distributed time with rate $\sigma+m_{q n} \beta+\xi \min \left(q_{q n}, m_{q n}\right)+\frac{j \gamma}{M_{q n}-m_{q n}}$. After a transition takes place, it will head to one of $\left(q_{q n}+1, m_{q n}\right),\left(q_{q n}, m_{q n}-1\right),\left(q_{q n}-1, m_{q n}\right),\left(q_{q n}, M_{q n}\right)$ with following probabilities:

$$
\begin{gathered}
P_{\left(q_{q n}, m_{q n}\right)\left(q_{q n}+1, m_{q n}\right)}(a=2)=\frac{\sigma}{\sigma+m_{q n} \beta+\xi \min \left(q_{q n}, m_{q n}\right)+\frac{j \gamma}{M_{q n}-m_{q n}}}, \\
P_{\left.\left(q_{q n}, m_{q n}\right)\left(q_{q n}, m_{q n}-1\right)\right)}(a=2)=\frac{m_{q n} \beta}{\sigma+m_{q n} \beta+\xi \min \left(q_{q n}, m_{q n}\right)+\frac{j \gamma}{M_{q n}-m_{q n}}}, \\
P_{\left(q_{q n}, m_{q n}\right)\left(q_{q n}-1, m_{q n}\right)}(a=2)=\frac{\xi \min \left(q_{q n}, m_{q n}\right)}{\sigma+m_{q n} \beta+\xi \min \left(q_{q n}, m_{q n}\right)+\frac{j \gamma}{M_{q n}-m_{q n}}}, \\
P_{\left(q_{q n} m_{q n}\right)\left(q_{q n}, M_{q n}\right)}(a=2)=\frac{\frac{j \gamma}{\sigma M_{q n}-m_{q n}}}{\sigma+m_{q n} \beta+\xi \min \left(q_{q n}, m_{q n}\right)+\frac{j \gamma}{M_{q n}-m_{q n}}} .
\end{gathered}
$$

Given the condition of next state is $\left(q_{q n}, m_{q n}\right)^{\prime}$, the duration until the transition from $\left(q_{q n}, m_{q n}\right)$ to $\left(q_{q n}, m\right)^{\prime}$ follows exponential distribution $F_{\left(q_{q n}, m_{q n}\right)\left(q_{q n}, m_{q n}\right)^{\prime}}(\cdot \mid a)$ :

$$
\begin{aligned}
& F_{\left(q_{q n}, m_{q n}\right)\left(\left(q q n_{q n}+1, m_{q n}\right)\right.}(t \mid a=1)=F_{\left(q_{q n}, m_{q n}\right)\left(q_{q n}, m_{q n}-1\right)}(t \mid a=1)=F_{\left(q_{q n}, m_{q n}\right)\left(q_{q n}-1, m_{q n}\right)}(t \mid a=1)=1-e^{-\left(\sigma+\xi \min \left(q_{q n}, m_{q n}\right)+m_{q n} \beta\right) t} \\
& F_{\left(q_{q n}, m_{q n}\right)\left(q_{q n}+1, m_{q n}\right)}(t \mid a=2)=F_{\left(q_{q n}, m_{q n}\right)\left(q_{q n}-1, m_{q n}\right)}(t \mid a=2)=F_{\left(q_{q n}, m_{q n}\right)\left(q_{q n}, m_{q n}-1\right)}(t \mid a=2)=F_{\left(q_{q n}, m_{q n}\right)\left(q_{q n}, M_{q n}\right)}(t \mid a=2) \\
& =1-e^{-\left(\sigma+\xi \min \left(q_{q n}, m_{q n}\right)+m_{q n} \beta+\frac{j \gamma}{M q n-m q n}\right) t}
\end{aligned}
$$

Finally, the cost structure of the model can be specified as follows.

If action $a$ is implemented in $\left(q_{q n}, m_{q n}\right)$, accordingly the direct cost $C\left(\left(q_{q n}, m_{q n}\right), a\right)$ is caused, and moreover, the cost rate $c\left(\left(q_{q n}, m_{q n}\right), a\right)$ is exerted till the latter transition arises:

$$
C\left(\left(q_{q n}, m_{q n}\right), a=1\right)=0, c\left(\left(q_{q n}, m_{q n}\right), a=1\right)=k q_{q n}, c\left(\left(q_{q n}, m_{q n}\right), a=1\right)=k q_{q n} .
$$


Furthermore, based on the above mechanism of transition, notice that the probability to complete the replacement is $\frac{\frac{j \gamma}{M_{q n}-m_{q n}}}{\alpha+\sigma\left(q_{q n}, m_{q n}\right)+\frac{j \gamma}{M q n-m q n}}$.

Therefore, the expected replacement cost of action $a=2$ is equal to

$$
C\left(\left(q_{q n}, m_{q n}\right), a=2\right)=\frac{\frac{j \gamma}{M_{q n}-m_{q n}}}{\alpha+\sigma\left(q_{q n}, m_{q n}\right)+\frac{j \gamma}{M_{q n}-m_{q n}}} *\left[F_{c}+\left(M_{q n}-m_{q n}\right) r_{c}\right],
$$

which represents the property that the replacement cost is caused unless the replacement is accomplished until the next transition. $F_{c}$ represents the fixed replacement cost for triggering replacements, $r_{c}$ indicates the variable replacement cost of one replaced server, and $k$ denotes the keeping cost per unit time for each product. If the transition happens for $t$ units of time, then the total cost will be $C\left(\left(q_{q n}, m_{q n}\right), a\right)+t^{*} c\left(\left(q_{q n}, m_{q n}\right), a\right)$.

\section{Formulation under the Discounted Cost Criterion}

It is assumed that costs are continuously discounted with rate $\alpha \geq 0$, and minimize the expected total discounted cost $T U_{\alpha}\left(q_{q n}, m_{q n}\right)$. Applying the principle of continuous time Markov Decision Process (MDP) [24], the proposed model can be converted into a discrete time MDP as follows:

The one-stage cost $\overline{C_{\alpha}}\left(\left(q_{q n}, m_{q n}\right), a\right)$ is derived as the following discrete time equation:

$$
\overline{C_{\alpha}}\left(\left(q_{q n}, m_{q n}\right), a\right)=C\left(\left(q_{q n}, m_{q n}\right), a\right)+\sum_{\left(q_{q n}, m_{q n}\right)^{\prime}} P_{\left(q_{q n}, m_{q n}\right)}\left(q_{q n}, m_{q n}\right)^{\prime}(a) \int_{0}^{\infty} \int_{0}^{t} e^{-\alpha s} \mathcal{C}\left(\left(q_{q n}, m_{q n}\right), a\right) d s d F_{\left(q_{q n}, m_{q n}\right)\left(q_{q n}, m_{q n}\right)^{\prime}}(t \mid a) .
$$

Specially, when $a=1$ (Proceed the production process), then

$$
\overline{C_{\alpha}}\left(\left(q_{q n}, m_{q n}\right), 1\right)=0+\int_{0}^{\infty} \int_{0}^{t} e^{-\alpha s} d s k q \sigma\left(q_{q n}, m_{q n}\right) e^{-\sigma\left(q_{q n}, m_{q n}\right) t} d t=\frac{k q_{q n}}{\alpha+\sigma\left(q_{q n}, m_{q n}\right)} .
$$

Also, when $a=2$ (Conduct the group replacement),

$$
\begin{aligned}
& \overline{C_{\alpha}}\left(\left(q_{q n}, m_{q n}\right), 2\right)=\frac{\frac{i \gamma}{M_{q n}-m_{q n}}}{\alpha+\sigma\left(q_{q n}, m_{q n}\right)+\frac{j \gamma}{M q n-m_{q n}}}\left[F+\left(M_{q n}-m_{q n}\right) r_{c}\right]+\int_{0}^{\infty} \int_{0}^{t} e^{-\alpha s} d s k q e^{-\left[\sigma\left(q_{q n}, m_{q n}\right)+\frac{j \gamma}{M q q n-m_{q n}}\right] t} d t \\
& =\frac{\frac{i \gamma}{M_{q n}-m_{q n}}}{\alpha+\sigma\left(q_{q n}, m_{q n}\right)+\frac{j \gamma}{M_{q n}-m_{q n}}}\left[F+\left(M_{q n}-m_{q n}\right) r_{c}\right]+\frac{k q_{q n}}{\alpha+\sigma\left(q_{q n}, m_{q n}\right)+\frac{j \gamma}{M_{q n}-m_{q n}}} .
\end{aligned}
$$

The future discounted cost function can be derived as follows:

$$
\overline{T U}\left(\left(q_{q n}, m_{q n}\right), a\right)=\sum_{\left(q q n, m_{q n}\right)^{\prime}} P_{\left(q_{q n}, m_{q n}\left(q_{q n}, m_{q n}\right)^{\prime}\right.}(a) \int_{0}^{\infty} e^{-\alpha t} T U_{\alpha}\left(\left(q_{q n}, m_{q n}\right)^{\prime}\right) d F_{\left(q_{q n}, m_{q n}\right)\left(q_{q n}, m_{q n}\right)^{\prime}}(t \mid a) .
$$

Specially, when $a=1$, through some simple intermediary integration:

$$
\begin{aligned}
& \overline{T U}\left(\left(q_{q n}, m_{q n}\right), 1\right)=\sum_{\left(q_{q n}, m_{q n}\right)^{\prime}} P_{\left(q_{q n}, m_{q n}\right)\left(q_{q n}, m_{q n}\right)^{\prime}}(1) \int_{0}^{\infty} e^{-\alpha t} T U_{\alpha}\left(\left(q_{q n}, m_{q n}\right)^{\prime}\right) \sigma\left(q_{q n}, m_{q n}\right) e^{-\sigma\left(q_{q n}, m_{q n}\right) t} d t \\
= & \sum_{\left(q_{q n}, m_{q n}\right)^{\prime}} P_{\left(q_{q n}, m_{q n}\right)\left(q_{q n}, m_{q n}\right)^{\prime}}(1) T U_{\alpha}\left(\left(q_{q n}, m_{q n}\right)^{\prime}\right)\left(\frac{\sigma\left(q_{q n}, m_{q n}\right)}{\alpha+\sigma\left(q_{q n}, m_{q n}\right)}\right) \\
= & \frac{\sigma}{\alpha+\sigma\left(q_{q n}, m_{q n}\right)} T U_{\alpha}\left(q_{q n}+1, m_{q n}\right)+\frac{m_{q n} \beta}{\alpha+\sigma\left(q_{q n}, m_{q n}\right)} T U_{\alpha}\left(q_{q n}, m_{q n}-1\right)+\frac{\zeta \min \left(q_{q n}, m_{q n}\right)}{\alpha+\sigma\left(q_{q n}, m_{q n}\right)} * \\
& T U_{\alpha}\left(q_{q n}-1, m_{q n}\right) .
\end{aligned}
$$


Similarly, when $a=2$,

$$
\begin{aligned}
& \overline{T U}\left(\left(q_{q n}, m_{q n}\right), 2\right) \\
& =\sum_{\left(q_{q n}, m_{q n}\right)^{\prime}} P_{\left(q_{q n}, m_{q n}\right)\left(q_{q n}, m_{q n}\right)^{\prime}}(1) \int_{0}^{\infty} e^{-\alpha t} T U_{\alpha}\left(\left(q_{q n}, m_{q n}\right)^{\prime}\right)\left[\sigma\left(q_{q n}, m_{q n}\right)+\frac{j \gamma}{M_{q n}-m_{q n}}\right] e^{-\left[\sigma\left(q_{q n}, m_{q n}\right)+\frac{j \gamma}{M q n-m q n}\right] t} d t \\
& =\sum_{\left(q_{q n}, m_{q n}\right)^{\prime}} P_{\left(q_{q n}, m_{q n}\right)\left(q_{q n}, m_{q n}\right)^{\prime}}(1) T U_{\alpha}\left(\left(q_{q n}, m_{q n}\right)^{\prime}\right)\left(\frac{\sigma\left(q_{q n}, m_{q n}\right)+\frac{j \gamma}{M q n-m_{q n}}}{\alpha+\sigma\left(q_{q n}, m_{q n}\right)+\frac{j \gamma}{M q n-m_{q n}}}\right) \\
& =\frac{\sigma}{\alpha+\sigma\left(q_{q n}, m_{q n}\right)+\frac{j \gamma}{M q n-m_{q n}}} * T U_{\alpha}\left(q_{q n}+1, m_{q n}\right)+\frac{m_{q n} \beta}{\alpha+\sigma\left(q_{q n}, m_{q n}\right)+\frac{j \gamma}{M q n-m_{q n}}} * T U_{\alpha}\left(q_{q n}, m_{q n}-1\right) \\
& +\frac{\zeta \min \left(q_{q n}, m_{q n}\right)}{\alpha+\sigma\left(q_{q n}, m_{q n}+\frac{j \gamma}{M_{q n}-m_{q n}}\right.} * T U_{\alpha}\left(q_{q n}-1, m_{q n}\right)+\frac{\frac{j \gamma}{M_{q n}-m_{q n}}}{\alpha+\sigma\left(q_{q n}, m_{q n}\right)+\frac{j \gamma}{M_{q n}-m_{q n}}} * T U_{\alpha}\left(q_{q n}, M_{q n}\right) \text {. }
\end{aligned}
$$

Based on the above outcomes, the discrete time version of optimality equations can be denoted as $T U_{\alpha}\left(q_{q n}, m_{q n}\right)=\min \left\{T U_{\alpha}^{1}\left(q_{q n}, m_{q n}\right), T U_{\alpha}^{2}\left(q_{q n}, m_{q n}\right)\right\}$, where

$$
\begin{aligned}
& T U_{\alpha}^{1}\left(q_{q n}, m_{q n}\right)=\overline{C_{\alpha}}\left(\left(q_{q n}, m_{q n}\right), 1\right)+\overline{T U}\left(\left(q_{q n}, m_{q n}\right), 1\right) \\
& =\frac{k q_{q n}}{\alpha+\sigma\left(q_{q n}, m_{q n}\right)}+\frac{\sigma}{\alpha+\sigma\left(q_{q n}, m_{q n}\right)} T U_{\alpha}\left(q_{q n}+1, m_{q n}\right)+\frac{m_{q n} \beta}{\alpha+\sigma\left(q_{q n}, m_{q n}\right)} T U_{\alpha}\left(q_{q n} m_{q n}-1\right)+\frac{\zeta \min \left(q_{q n}, m_{q n}\right)}{\alpha+\sigma\left(q_{q n}, m_{q n}\right)} * \\
& T U_{\alpha}\left(q_{q n}-1, m_{q n}\right) \text {. } \\
& T U_{\alpha}^{2}\left(q_{q n}, m_{q n}\right)=\frac{\frac{j \gamma}{M_{q n}-m_{q n}}}{\alpha+\sigma\left(q_{q n}, m_{q n}\right)+\frac{j \gamma}{M_{q n}-m_{q n}}}\left[F+\left(M_{q n}-m_{q n}\right) r_{c}\right]+\frac{k q_{q n}}{\alpha+\sigma\left(q_{q n}, m_{q n}\right)+\frac{j \gamma}{M_{q n}-m_{q n}}}+ \\
& \frac{\sigma}{\alpha+\sigma\left(q_{q n}, m_{q n}\right)+\frac{j \gamma}{M_{q n}-m_{q n}}} T U_{\alpha}\left(q_{q n}+1, m_{q n}\right)+\frac{m_{q n} \beta}{\alpha+\sigma\left(q_{q n}, m_{q n}\right)+\frac{j \gamma}{M_{q n}-m_{q n}}} T U_{\alpha}\left(q_{q n}, m_{q n}-1\right)+ \\
& \frac{\zeta \min \left(q_{q n}, m_{q n}\right)}{\alpha+\sigma\left(q_{q n}, m_{q n}\right)+\frac{j \gamma}{M_{q n}-m_{q n}}} T U_{\alpha}\left(q_{q n}-1, m_{q n}\right)+\frac{\frac{j \gamma}{M_{q n}-m_{q n}}}{\alpha+\sigma\left(q_{q n}, m_{q n}\right)+\frac{j \gamma}{M_{q n}-m_{q n}}} T U_{\alpha}\left(q_{q n}, M_{q n}\right) \text {. }
\end{aligned}
$$

The state space is not countable and the one-stage cost $\bar{C}\left(\left(q_{q n}, m_{q n}\right), a\right)$ is unbounded. Applying standard results from unbounded cost MDPs [26] (Th. 4.2.3, Prop. 4.3.1, (c)), the subsequent Lemma can be obtained:

\section{Lemma 1.}

(i) $T U_{\alpha}\left(q_{q n}, m_{q n}\right)$ is the sole solution to the equations of optimality in (5).

(ii) There exists an optimal stationary policy, which conducts action to minimize the right side of Equation (5).

(iii) Assume $T U_{\alpha}\left(q_{q n}, m_{q n} ; n=0\right)=0$,

$$
T U_{\alpha}\left(q_{q n}, m_{q n} ; n\right)=\min _{a \in A\left(q_{q n}, m_{q n}\right)}\left\{\bar{C}\left(\left(q_{q n}, m_{q n}\right), a\right)+\overline{T U}\left(\left(q_{q n}, m_{q n}\right), a ; n-1\right)\right\} .
$$

Consequently, $\lim _{n \rightarrow \infty} T U_{\alpha}\left(q_{q n}, m_{q n} ; n\right)=T U_{\alpha}\left(q_{q n}, m_{q n}\right)$ for any preliminary state $\left(q_{q n}, m_{q n}\right)$.

\section{The Modified Uniformization Model}

Unfortunately, the discounted cost Equation (5) developed in Section 3 is very difficult to prove that there exists a threshold of amount of customer to trigger the group replacement based on different amount of operational servers. Therefore, this proposed IoT conditioned-based group replacement decision support system further revises the original discounted cost model into an equivalent model to facilitate the proof procedure by applying the uniformization approach. Several significant theoretical properties are proven and many numerical examples are conducted for two kinds of group replacement policies, respectively.

Section 3 shows that all transition rates are limited by $\alpha+\sigma+M_{q n} \beta+M_{q n} \zeta+j \gamma$ in state $\left(q_{q n}, m_{q n}\right)$. In this section, the uniformization approach proposed by $[27,28]$ is carried out to convert the proposed 
model in Section 3 into a similar model where all exponentially distributed sojourn times abide by the identical rate $\bar{\sigma} \geq \alpha+\sigma+M_{q n} \beta+M_{q n} \zeta+j \gamma$. This model can be modified as follows:

All transitions from each state happen at a common rate $\bar{\sigma}$. While the process stays in $\left(q_{q n}, m_{q n}\right)$, however, only a fraction $\frac{\sigma\left(q_{q n}, m_{q n}\right)}{\bar{\sigma}}$ are those transitions from $\left(q_{q n}, m_{q n}\right)$ to state $\left(q_{q n}, m_{q n}\right)^{\prime}$ not equal to $\left(q_{q n}, m_{q n}\right)$ and the others are transitions returning to $\left(q_{q n}, m_{q n}\right)$, which are called virtual transitions. Specially, we derive a similar discrete-time Markov chain in which transitions are described as follows:

(1) When $a=1$ (proceed the production process)

$\left(q_{q n}, m_{q n}\right) \rightarrow\left(q_{q n}+1, m_{q n}\right)$ with probability $\frac{\sigma}{\bar{\sigma}},\left(q_{q n}, m_{q n}\right) \rightarrow\left(q_{q n}, m_{q n}-1\right)$ with probability $\frac{m \beta}{\bar{\sigma}}$, $\left(q_{q n}, m_{q n}\right) \rightarrow\left(q_{q n}-1, m_{q n}\right)$ with probability $\frac{\zeta \min \left(q_{q n}, m_{q n}\right)}{\bar{\sigma}},\left(q_{q n}, m_{q n}\right) \rightarrow$ absorbing with probability $\frac{\alpha}{\bar{\sigma}},\left(q_{q n}, m_{q n}\right) \rightarrow\left(q_{q n}, m_{q n}\right)$ with probability $\left(1-\frac{\alpha+\sigma\left(q_{q n}, m_{q n}\right)}{\bar{\sigma}}\right)$.

(2) When $a=2$ (conduct the group replacement)

$\left(q_{q n}, m_{q n}\right) \rightarrow\left(q_{q n}+1, m_{q n}\right)$ with probability $\frac{\sigma}{\bar{\sigma}},\left(q_{q n}, m_{q n}\right) \rightarrow\left(q_{q n}, m_{q n}-1\right)$ with probability $\frac{m_{q n} \beta}{\bar{\sigma}},\left(q_{q n}, m_{q n}\right) \rightarrow\left(q_{q n}-1, m_{q n}\right)$ with probability $\frac{\zeta \min \left(q_{q n}, m_{q n}\right)}{\bar{\sigma}},\left(q_{q n}, m_{q n}\right) \rightarrow\left(q_{q n}, M_{q n}\right)$ with probability $\left.\frac{\frac{j \gamma}{M_{q n}-m_{q n}}}{\bar{\sigma}},\left(q_{q n}, m_{q n}\right) m_{q n}\right) \rightarrow$ absorbing state with probability $\frac{\alpha}{\bar{\sigma}},\left(q_{q n}, m_{q n}\right) \rightarrow\left(q_{q n}, m_{q n}\right)$ with probability $\left(1-\frac{\alpha+\frac{j \beta}{M_{q n}-m_{q n}}+\sigma\left(q_{q n}, m_{q n}\right)}{\bar{\sigma}}\right)$.

By applying this modified Markov decision process, the equation of optimality can be converted as follows:

Recollect the optimality Equation (5), while action $a=1, T U_{\alpha}\left(q_{q n}, m_{q n}\right)=U_{\alpha}^{1}\left(q_{q n}, m_{q n}\right)$ is acquired. By multiplying both parts of the equation with $\frac{\alpha+\sigma\left(q_{q n}, m_{q n}\right)}{\bar{\sigma}}$, then increasing both parts of the above equation with $V_{\alpha}\left(q_{q n}, m_{q n}\right)$ and moving $\frac{\alpha+\sigma\left(q_{q n}, m_{q n}\right)}{\bar{\sigma}} T U_{\alpha}\left(q_{q n}, m_{q n}\right)$ from left part of equation to right part of equation, we have

$$
\begin{aligned}
T U_{\alpha}^{1}\left(q_{q n}, m_{q n}\right) & =\frac{k q_{q n}}{\bar{\sigma}}+\frac{\sigma}{\bar{\sigma}} T U_{\alpha}\left(q_{q n}+1, m_{q n}\right)+\frac{m_{q n} \beta}{\bar{\sigma}} U_{\alpha}\left(q_{q n}, m_{q n}-1\right)+\frac{\zeta \min \left(q_{q n}, m_{q n}\right)}{\bar{\sigma}} T U_{\alpha}\left(q_{q n}-1, m_{q n}\right) \\
+ & \left(1-\frac{\alpha+\sigma\left(q_{q n}, m_{q n}\right)}{\bar{\sigma}}\right) T U_{\alpha}\left(q_{q n}, m_{q n}\right) .
\end{aligned}
$$

While action $a=2, T U_{\alpha}\left(q_{q n}, m_{q n}\right)=T U^{2}\left(q_{q n}, m_{q n}\right)$ is obtained. Through multiplying both parts of the above equation with $\frac{\alpha+\sigma\left(q_{q n}, m_{q n}\right)+\frac{j \gamma}{M_{q n}-m_{q n}}}{\bar{\sigma}}$, next adding both parts of the above equation with $T U_{\alpha}\left(q_{q n}, m_{q n}\right)$ and moving $\frac{\alpha+\sigma\left(q_{q n}, m_{q n}\right)+\frac{j \gamma}{M_{q n}-m_{q n}}}{\bar{\sigma}} T U_{\alpha}\left(q_{q n}, m_{q n}\right)$.

From left part of equation to right part of equation, we obtain

$$
\begin{aligned}
& T U_{\alpha}^{2}\left(q_{q n}, m_{q n}\right)=\frac{\frac{j \gamma}{M_{q n}-m_{q n}}}{\bar{\sigma}}\left[F+\left(M_{q n}-m_{q n}\right) r_{c}\right]+\frac{k q_{q n}}{\bar{\sigma}}+\frac{\sigma}{\bar{\sigma}} T U_{\alpha}\left(q_{q n}+1, m_{q n}\right)+\frac{m_{q n} \beta}{\bar{\sigma}} T U_{\alpha}\left(q_{q n}, m_{q n}-1\right)+ \\
& \frac{\zeta \min \left(q_{q n}, m_{q n}\right)}{\bar{\sigma}} T U_{\alpha}\left(q_{q n}-1, m_{q n}\right)+\frac{\overline{j \gamma}}{\overline{M_{q n}-m_{q n}}} T U_{\alpha}\left(q_{q n}, M_{q n}\right)+\left(1-\frac{\alpha+\sigma\left(q_{q n}, m_{q n}\right)+\frac{\bar{j} \gamma}{M_{q n}-m_{q n}}}{\bar{\sigma}}\right) U_{\alpha}\left(q_{q n} \cdot m_{q n}\right) .
\end{aligned}
$$

According to the outcomes of (6) and (7), the modified uniformization optimality equations can be denoted as

$$
T U_{\alpha}^{U}\left(q_{q n}, m_{q n}\right)=\min \left\{T U_{\alpha}^{1}\left(q_{q n}, m_{q n}\right), T U_{\alpha}^{2}\left(q_{q n}, m_{q n}\right)\right\}
$$

where

$$
\begin{aligned}
& T U_{\alpha}^{1}\left(q_{q n}, m_{q n}\right)=\frac{k q_{q n}}{\bar{\sigma}}+\frac{\sigma}{\bar{\sigma}} T U_{\alpha}\left(q_{q n}+1, m_{q n}\right)+\frac{m_{q n} \beta}{\bar{\sigma}} T U_{\alpha}\left(q_{q n}, m_{q n}-1\right)+\frac{\zeta \min \left(q_{q n}, m_{q n}\right)}{\bar{\sigma}} T U_{\alpha}\left(q_{q n}-1, m_{q n}\right) \\
& +\left(1-\frac{\alpha+\sigma\left(q_{q n}, m_{q n}\right)}{\bar{\sigma}}\right) T U_{\alpha}\left(q_{q n}, m_{q n}\right) . \\
& T U_{\alpha}^{2}\left(q_{q n}, m_{q n}\right)=\frac{\frac{j \gamma}{M q n-m q n}}{\bar{\sigma}}\left[F+\left(M_{q n}-m_{q n}\right) r_{c}\right]+\frac{k q_{q n}}{\bar{\sigma}}+\frac{\sigma}{\bar{\sigma}} T U_{\alpha}\left(q_{q n}+1, m_{q n}\right)+\frac{m_{q n} \beta}{\bar{\sigma}} T U_{\alpha}\left(q_{q n}, m_{q n}-1\right)+ \\
& \frac{\zeta \min \left(q_{q n}, m_{q n}\right)}{\bar{\sigma}} T U_{\alpha}\left(q_{q n}-1, m_{q n}\right)+\frac{\overline{j \gamma} M_{q n}-m_{q n}}{\bar{\sigma}} T U_{\alpha}\left(q_{q n}, M_{q n}\right)+\left(1-\frac{\alpha+\sigma\left(q_{q n}, m_{q n}\right)+\frac{j \gamma}{M_{q n}-m_{q n}}}{\bar{\lambda}}\right) T U_{\alpha}\left(q_{q n} \cdot m_{q n}\right) .
\end{aligned}
$$


Actually, the optimality equations in (5) and (8) are similar.

In the continuation Equation (8) will be applied to verify properties of the total cost function and the optimal replacement policy. Now the sequential approximated Equation (8) is described as follows:

$$
\begin{aligned}
& T U_{\alpha}\left(q_{q n}, m_{q n} ; 0\right)=0, \\
& T U_{\alpha}\left(q_{q n}, m_{q n} ; n\right)=\min \left\{\frac{k q_{q n}}{\bar{\sigma}}+\frac{\sigma}{\bar{\sigma}} T U_{\alpha}\left(q_{q n}+1, m_{q n} ; n-1\right)+\frac{m_{q n} \beta}{\bar{\sigma}} T U_{\alpha}\left(q_{q n}, m_{q n}-1 ; n-1\right)+\right. \\
& \frac{\zeta \min \left(q_{q n}, m_{q n}\right)}{\bar{\sigma}} T U_{\alpha}\left(q_{q n}-1 m_{q n} ; n-1\right)+\left(1-\frac{\alpha+\sigma\left(q_{q n}, m_{q n}\right)}{\bar{\sigma}}\right) T U_{\alpha}\left(q_{q n}, m_{q n} ; n-1\right), \\
& \frac{\frac{j \gamma}{M q n-m q n}}{\bar{\sigma}}\left[F+\left(M_{q n}-m_{q n}\right) r_{c}\right]+\frac{k q_{q n}}{\bar{\sigma}}+\frac{\sigma}{\bar{\sigma}} T U_{\alpha}\left(q_{q n}+1, m_{q n} ; n-1\right)+\frac{m_{q n} \beta}{\bar{\sigma}} T U_{\alpha}\left(q_{q n}, m_{q n}-1 ; n-1\right)+ \\
& \frac{\zeta \min \left(q_{q n}, m_{q n}\right)}{\bar{\sigma}} T U_{\alpha}\left(q_{q n}-1, m_{q n} ; n-1\right)+\frac{\frac{j \gamma}{M_{q n}-m_{q n}}}{\bar{\sigma}} T U_{\alpha}\left(q_{q n}, M_{q n} ; n-1\right)+ \\
& \left(1-\frac{\alpha+\sigma\left(q_{q n}, m_{q n}\right)+\frac{j \gamma}{M q n-m q n}}{\bar{\sigma}}\right) T U_{\alpha}\left(q_{q n}, m_{q n} ; n-1\right) .
\end{aligned}
$$

\section{The Theoretical Properties of Optimal Policy}

In this section, we establish some monotonicity properties for the total expected cost function $T U_{\alpha}\left(q_{q n}, m_{q n}\right)$ of the modified model, which will be applied to construct the framework of corresponding optimal policies. The next theorem shows the monotonicity of the total expected cost function on the number of customer in system.

Theorem 1. $T U_{\alpha}\left(q_{q n}, m_{q n}\right)$ is an increasing function of $q_{q n}$, the amount of customers in system.

Proof. See Appendix A.

With the result we have in Theorem 1 , we can derive the following Theorem about the monotonicity property of the total expected cost function on the number of working machines. First, the monotonicity properties for the difference function between $T U_{\alpha}^{1}\left(q_{q n}, m_{q n}\right)$ and $T U_{\alpha}^{2}\left(q_{q n}, m_{q n}\right)$ will be derived. These properties are important for proving the threshold structure of the optimal policy. Before doing so, we need to prove the following Lemma 2.

Lemma 2. $T U_{\alpha}\left(q_{q n}+1, m_{q n} ; n\right)-T U_{\alpha}\left(q_{q n}, m_{q n} ; n\right) \leq \frac{k}{\alpha}$ for all $\left(q_{q n}, m_{q n}\right)$.

Proof. It is noted that $T U_{\alpha}\left(q_{q n}, m_{q n} ; n\right)$ indicates the minimum expected discounted cost for an $n$-period group replacement problem, in which $n$ transitions are permitted till the finish of the horizon. The difference between $T U_{\alpha}\left(q_{q n}+1, m_{q n} ; n\right)$ and $T U_{\alpha}\left(q_{q n}, m_{q n} ; n\right)$ is that one more customer present in $T U_{\alpha}\left(q_{q n}+1, m_{q n} ; n\right)$ than $T U_{\alpha}\left(q_{q n}, m_{q n} ; n\right)$. Thus, an upper bound for the difference $T U_{\alpha}\left(q_{q n}+1, m_{q n} ; n\right)-T U_{\alpha}\left(q_{q n}, m_{q n} ; n\right)$ is provided by the total discounted keeping cost incurred because of that one more customer within $n$ transitions. If the additional customer stays in system for all $n$ transitions, in the light of the uniformization model, the time between transitions complies with exponential distribution with rate $\bar{\sigma}$. Thus, the total time $T_{n}$ follows a Gamma distribution with parameters $(n, \bar{\sigma})$ :

$$
f(t)=\left\{\begin{array}{c}
\frac{\bar{\sigma}^{n} t^{n-1} e^{-\bar{\sigma} t}}{(n-1) !} \quad t \geq 0 \\
0 \quad t<0
\end{array} .\right.
$$

Consequently, the expected discounted keeping cost can be denoted as:

$$
E\left[k \int_{0}^{t} e^{\alpha s} d s\right]=k \int_{0}^{\infty} \int_{0}^{t} e^{-\alpha s} d s f(t) d t=k \int_{0}^{\infty} \frac{1}{\alpha}\left(1-e^{-\alpha t}\right) f(t) d t=\frac{k}{\alpha}\left[1-\int_{0}^{\infty} e^{-\alpha t} f(t) d t\right] \leq \frac{k}{\alpha} .
$$

The proof is completed.

Theorem 2. $T U_{\alpha}\left(q_{q n}, m_{q n}\right)-T U_{\alpha}\left(q_{q n}, M_{q n}\right)$ is increasing in $q_{q n}$. 
Proof. See Appendix A.

Theorem 3. $T U_{\alpha}^{1}\left(q_{q n}, m_{q n}\right)-T U_{\alpha}^{2}\left(q_{q n}, m_{q n}\right)$ is increasing in $q_{q n}$.

Proof. The proof will be conducted by induction on $n$. From (9), for $n=1$, we have

$$
T U_{\alpha}^{1}\left(q_{q n}, m_{q n} ; n=1\right)-T U_{\alpha}^{2}\left(q_{q n}, m_{q n} ; n=1\right)=-\frac{\frac{j \gamma}{M_{q n}-m_{q n}}}{\bar{\sigma}}\left[F+\left(M_{q n}-m_{q n}\right) r_{c}\right] .
$$

It is obvious that $T U_{\alpha}^{1}\left(q_{q n}, m_{q n} ; n=1\right)-T U_{\alpha}^{2}\left(q_{q n}, m_{q n} ; n=1\right)$ is non-decreasing in $q_{q n}$.

According to the above outcome, it can be assumed that $T U_{\alpha}^{1}\left(q_{q n}, m_{q n} ; n\right)-T U_{\alpha}^{2}\left(q_{q n}, m_{q n} ; n\right)$ is increasing in $q_{q n}$, then it need to be proved $T U_{\alpha}^{1}\left(q_{q n}, m_{q n} ; n+1\right)-T U_{\alpha}^{2}\left(q_{q n}, m_{q n} ; n+1\right)$ is also increasing in $q_{q n}$. From (9), we have

$$
\begin{aligned}
& \operatorname{TU}_{\alpha}^{1}\left(q_{q n}, m_{q n} ; n+1\right)-U_{\alpha}^{2}\left(q_{q n}, m_{q n} ; n+1\right) \\
& =-\frac{\frac{j \gamma}{M_{q n}-m_{q n}}}{\bar{\sigma}}\left[F+\left(M_{q n}-m_{q n}\right) r_{c}\right]+\frac{\frac{j \gamma}{M_{q n}-m_{q n}}}{\bar{\sigma}}\left[T U_{\alpha}\left(q_{q n}, m_{q n} ; n\right)-T U_{\alpha}\left(q_{q n}, M_{q n} ; n\right)\right] .
\end{aligned}
$$

According the above result and Theorem 2, it can be concluded that $T U_{\alpha}^{1}\left(q_{q n}, m_{q n} ; n+1\right)-$ $T U_{\alpha}^{2}\left(q_{q n}, m_{q n} ; n+1\right)$ is increasing in $q_{q n}$.

Based on regular theses applying successive approximations of discounted MDPs, $T U_{\alpha}^{1}\left(q_{q n}, m_{q n}\right)-$ $T U_{\alpha}^{2}\left(q_{q n}, m_{q n}\right)=\lim _{n}\left\{T U_{\alpha}^{1}\left(q_{q n}, m_{q n} ; n\right)-T U_{\alpha}^{2}\left(q_{q n}, m_{q n} ; n\right)\right\}$ is increasing in $q_{q n}$. The proof is thus carried through.

Now we present the optimal policy for this model.

Theorem 4. There exist thresholds $q_{q n}^{*}\left(m_{q n}\right) \geq 0$, in which an $\alpha$-optimal policy is do the group replacement if $q_{q n} \geq q_{q n}^{*}(m)$ while the system is in state $\left(q_{q n}, m_{q n}\right)$.

Proof. According to (8), it can be obtained that

$$
\begin{aligned}
& T U_{\alpha}\left(q_{q n}, m_{q n}\right)=\min \left\{\frac{k q_{q n}}{\bar{\sigma}}+\frac{\sigma}{\bar{\sigma}} T U_{\alpha}\left(q_{q n}+1, m_{q n}\right)+\frac{m_{q n} \beta}{\bar{\sigma}} T U_{\alpha}\left(q_{q n}, m_{q n}-1\right)+\frac{\zeta \min \left(q_{q n}, m_{q n}\right)}{\bar{\sigma}} *\right. \\
& T U_{\alpha}\left(q_{q n}-1, m_{q n}\right)+\left(1-\frac{\alpha+\sigma\left(q_{q n}, m_{q n}\right)}{\bar{\sigma}}\right) T U_{\alpha}\left(q_{q n}, m_{q n}\right), \frac{\overline{M_{q n}-m_{q n}}}{\bar{\sigma}}\left[F+\left(M_{q n}-m_{q n}\right) r_{c}\right] \\
& +\frac{k q_{q n}}{\bar{\sigma}}+\frac{\sigma}{\bar{\sigma}} T U_{\alpha}\left(q_{q n}+1, m_{q n}\right)+\frac{m_{q n} \beta}{\bar{\sigma}} T U_{\alpha}\left(q_{q n}, m_{q n}-1\right)+\frac{\zeta \min \left(q_{q n}, m_{q n}\right)}{\bar{\sigma}} T U_{\alpha}\left(q_{q n}-1, m_{q n}\right)+ \\
& \frac{\overline{M_{q n}-m_{q n}}}{\bar{\sigma}} T U_{\alpha}\left(q_{q n}, M_{q n}\right)+\left(1-\frac{\alpha+\sigma\left(q_{q n}, m_{q n}\right)+\frac{j \gamma}{M_{q n}-m_{q n}}}{\bar{\lambda}}\right) T U_{\alpha}\left(q_{q n}, m_{q n}\right) .
\end{aligned}
$$

Let

$$
\begin{aligned}
& q_{q n}^{*}\left(m_{q n}\right)=\min \left\{q_{q n}: \frac{k q_{q n}}{\bar{\sigma}}+\frac{\sigma}{\bar{\sigma}} T U_{\alpha}\left(q_{q n}+1, m_{q n}\right)+\frac{m_{q n} \beta}{\bar{\sigma}} T U_{\alpha}\left(q_{q n}, m_{q n}-1\right)+\frac{\zeta \min \left(q_{q n}, m_{q n}\right)}{\bar{\sigma}} *\right. \\
& T U_{\alpha}\left(q_{q n}-1, m_{q n}\right)+\left(1-\frac{\alpha+\sigma\left(q_{q n}, m_{q n}\right)}{\bar{\sigma}}\right) T U_{\alpha}\left(q_{q n}, m_{q n}\right)>\frac{\frac{j \gamma}{M_{q n}-m_{q n}}}{\bar{\sigma}}\left[F+\left(M_{q n}-m_{q n}\right) r_{c}\right]+\frac{k q_{q n}}{\bar{\sigma}}+ \\
& \frac{\sigma}{\bar{\sigma}} T U_{\alpha}\left(q_{q n}+1, m_{q n}\right)+\frac{m_{q n} \beta}{\bar{\sigma}} T U_{\alpha}\left(q_{q n}, m_{q n}-1\right)+\frac{\zeta \min \left(q_{q n}, m_{q n}\right)}{\bar{\sigma}} * T U_{\alpha}\left(q_{q n}-1, m_{q n}\right) \\
& \left.+\frac{\frac{j \gamma}{M_{q n}-m_{q n}}}{\bar{\sigma}} T U_{\alpha}\left(q_{q n}, M_{q n}\right)+\left(1-\frac{\left.\alpha+\sigma\left(q_{q n}, m_{q n}\right)+\frac{j \gamma}{M_{q n}-m_{q n}}\right)}{\bar{\sigma}}\right) T U_{\alpha}\left(q_{q n}, m_{q n}\right)\right\} .
\end{aligned}
$$

Now, according to Theorem 3 , it confirms that $T U_{\alpha}^{1}\left(q_{q n}, m_{q n}\right)-T U_{\alpha}^{2}\left(q_{q n}, m_{q n}\right)$ is increasing in $q_{q n}$, and thus it can be obtained that

$$
T U_{\alpha}\left(q_{q n}, m_{q n}\right)=\left\{\begin{array}{l}
T U_{\alpha}^{1}\left(q_{q n}, m_{q n}\right) \text { for } q_{q n}<q_{q n}^{*}\left(m_{q n}\right) \\
T U_{\alpha}^{2}\left(q_{q n}, m_{q n}\right) \text { for } q_{q n} \geq q_{q n}^{*}(m)
\end{array},\right.
$$


where

$$
\begin{aligned}
& T U_{\alpha}^{1}\left(q_{q n}, m_{q n}\right)=\frac{k q_{q n}}{\bar{\sigma}}+\frac{\sigma}{\bar{\sigma}} T U_{\alpha}\left(q_{q n}+1, m_{q n}\right)+\frac{m_{q n} \beta}{\bar{\sigma}} T U_{\alpha}\left(q_{q n}, m_{q n}-1\right)+\frac{\zeta \min \left(q_{q n}, m_{q n}\right)}{\bar{\sigma}} T U_{\alpha}\left(q_{q n}-1, m_{q n}\right) \\
& +\left(1-\frac{\alpha+\sigma\left(q_{q n}, m_{q n}\right)}{\bar{\sigma}}\right) T U_{\alpha}\left(q_{q n}, m_{q n}\right) . \\
& T U_{\alpha}^{2}\left(q_{q n}, m_{q n}\right)=\frac{\frac{j \gamma}{M q n}-m_{q n}}{\bar{\sigma}}\left[F+\left(M_{q n}-m_{q n}\right) r_{c}\right]+\frac{k q q n}{\bar{\sigma}}+\frac{\sigma}{\bar{\sigma}} T U_{\alpha}\left(q_{q n}+1, m_{q n}\right)+\frac{m_{q n} \beta}{\bar{\sigma}} T U_{\alpha}\left(q_{q n}, m_{q n}-1\right) \\
& +\frac{\zeta \min \left(q_{q n}, m_{q n}\right)}{\bar{\sigma}} T U_{\alpha}\left(q_{q n}-1, m_{q n}\right)+\frac{\frac{i \gamma}{M_{q n}-m_{q n}}}{\bar{\sigma}} T U_{\alpha}\left(q_{q n}, M_{q n}\right)+\left(1-\frac{\alpha+\sigma\left(q_{q n}, m_{q n}\right)+\frac{j \gamma}{M q n-m q n}}{\bar{\sigma}}\right) * \\
& T U_{\alpha}\left(q_{q n}, m_{q n}\right) .
\end{aligned}
$$

The proof is hence completed.

\section{Numerical Results}

This section conducts different numerical examples to demonstrate the related theorems and lemmas, which have been proved previously in Section 5. Several numerical examples were programmed in Maple 10 (Version 10, Maplesoft, Waterloo, ON, Canada) and were run on Asus TX201L (Asus, Taipei, Taiwan) with Intel(R) Core(TM) i7-4500U CPU (Intel Corporation, Santa Clara, CA, USA)@ 2.40GHz, 4.00 GB RAM.

First, in Example 1, the total discounted costs acquired from the successive approximation version

\begin{tabular}{|c|c|c|c|c|c|c|c|c|c|}
\hline$m$ & 0 & 1 & 2 & 3 & 4 & 5 & 6 & 7 & 8 \\
\hline \multicolumn{10}{|l|}{$q=0$} \\
\hline init & 82.843 & 73.144 & 66.434 & 61.573 & 57.924 & 55.100 & 52.859 & 51.042 & 49.540 \\
\hline unif & 81.696 & 71.998 & 65.288 & 60.428 & 56.778 & 53.953 & 51.712 & 49.895 & 48.393 \\
\hline dev & 1.147 & 1.146 & 1.146 & 1.145 & 1.146 & 1.147 & 1.147 & 1.147 & 1.147 \\
\hline dev.\% & $1.4 \%$ & $1.59 \%$ & $1.76 \%$ & $1.86 \%$ & $1.98 \%$ & $2.08 \%$ & $2.17 \%$ & $2.25 \%$ & $2.32 \%$ \\
\hline \multicolumn{10}{|l|}{$q=1$} \\
\hline init & 96.097 & 82.907 & 74.379 & 68.509 & 64.272 & 61.092 & 58.627 & 56.664 & 55.065 \\
\hline unif & 94.951 & 81.761 & 73.233 & 67.363 & 63.126 & 59.946 & 57.480 & 55.517 & 53.918 \\
\hline dev & 1.146 & 1.146 & 1.146 & 1.146 & 1.146 & 1.146 & 1.147 & 1.147 & 1.147 \\
\hline dev.\% & $1.19 \%$ & $1.38 \%$ & $1.54 \%$ & $1.67 \%$ & $1.78 \%$ & $1.88 \%$ & $1.96 \%$ & $2.02 \%$ & $2.08 \%$ \\
\hline \multicolumn{10}{|l|}{$q=2$} \\
\hline init & 107.486 & 95.392 & 83.636 & 76.170 & 70.975 & 67.282 & 64.510 & 62.355 & 60.633 \\
\hline unif & 106.339 & 94.245 & 82.490 & 74.964 & 69.829 & 66.136 & 63.363 & 61.208 & 59.486 \\
\hline dev & 1.147 & 1.147 & 1.146 & 1.206 & 1.146 & 1.146 & 1.147 & 1.147 & 1.147 \\
\hline dev.\% & $1.07 \%$ & $1.20 \%$ & $1.37 \%$ & $1.58 \%$ & $1.61 \%$ & $1.70 \%$ & $1.78 \%$ & $1.84 \%$ & $1.89 \%$ \\
\hline \multicolumn{10}{|l|}{$q=3$} \\
\hline init & 119.025 & 107.727 & 95.423 & 84.892 & 78.267 & 73.783 & 70.564 & 68.145 & 66.259 \\
\hline unif & 117.878 & 106.580 & 94.277 & 83.746 & 77.121 & 72.636 & 69.418 & 66.998 & 65.112 \\
\hline dev & 1.147 & 1.147 & 1.146 & 1.146 & 1.146 & 1.147 & 1.146 & 1.147 & 1.147 \\
\hline dev.\% & $0.96 \%$ & $1.06 \%$ & $1.2 \%$ & $1.34 \%$ & $1.46 \%$ & $1.55 \%$ & $1.62 \%$ & $1.68 \%$ & $1.73 \%$ \\
\hline \multicolumn{10}{|l|}{$q=4$} \\
\hline init & 130.775 & 119.232 & 107.915 & 95.965 & 86.616 & 80.804 & 76.890 & 74.083 & 71.970 \\
\hline unif & 129.629 & 118.085 & 106.769 & 94.819 & 85.470 & 79.657 & 75.743 & 72.937 & 70.823 \\
\hline dev & 1.146 & 1.147 & 1.146 & 1.146 & 1.146 & 1.147 & 1.147 & 1.146 & 1.147 \\
\hline dev.\% & $0.88 \%$ & $0.96 \%$ & $1.06 \%$ & $1.19 \%$ & $1.32 \%$ & $1.42 \%$ & $1.49 \%$ & $1.54 \%$ & $1.59 \%$ \\
\hline
\end{tabular}
of initial optimality Equation (5) is compared with the modified optimality equation of uniformization (8) by running 300 iterations and the outcomes are presented in the following Table 1.

Table 1. Total discounted costs of initial and modified optimality equation. $M=8, \sigma_{m}=5, m=0, \ldots, 8$, $\zeta=3, \beta=1, F=25, r_{c}=2, k=20, \gamma=4, j=4, \alpha=0.8$, iteration $=300$. 
Table 1. Cont

\begin{tabular}{cccccccccc}
\hline $\boldsymbol{m}$ & $\mathbf{0}$ & $\mathbf{1}$ & $\mathbf{2}$ & $\mathbf{3}$ & $\mathbf{4}$ & $\mathbf{5}$ & $\mathbf{6}$ & $\mathbf{7}$ & $\mathbf{8}$ \\
\hline$q=5$ & & & & & & & & & \\
init & 142.822 & 130.681 & 119.645 & 108.079 & 97.032 & 88.771 & 83.677 & 80.259 & 77.809 \\
unif & 141.675 & 129.534 & 118.498 & 106.933 & 95.886 & 87.625 & 82.530 & 79.113 & 76.663 \\
dev & 1.147 & 1.147 & 1.147 & 1.146 & 1.146 & 1.146 & 1.147 & 1.146 & 1.146 \\
dev.\% & $0.80 \%$ & $0.88 \%$ & $0.96 \%$ & $1.06 \%$ & $1.18 \%$ & $1.29 \%$ & $1.37 \%$ & $1.43 \%$ & $1.47 \%$ \\
\hline$q=6$ & & & & & & & & & \\
init & 155.278 & 142.372 & 131.028 & 120.239 & 108.719 & 98.615 & 91.313 & 86.845 & 83.858 \\
unif & 154.131 & 141.225 & 129.881 & 119.093 & 107.573 & 97.469 & 90.167 & 85.698 & 82.711 \\
dev & 1.147 & 1.147 & 1.147 & 1.146 & 1.146 & 1.146 & 1.146 & 1.147 & 1.147 \\
dev.\% & $0.73 \%$ & $0.81 \%$ & $0.88 \%$ & $0.95 \%$ & $1.05 \%$ & $1.16 \%$ & $1.26 \%$ & $1.32 \%$ & $1.37 \%$ \\
\hline$q=7$ & & & & & & & & & \\
init & 168.291 & 154.506 & 142.537 & 131.777 & 120.946 & 109.821 & 100.651 & 94.193 & 90.270 \\
unif & 167.144 & 153.359 & 141.391 & 130.631 & 119.800 & 108.674 & 99.505 & 93.047 & 89.123 \\
dev & 1.147 & 1.147 & 1.146 & 1.146 & 1.146 & 1.147 & 1.146 & 1.146 & 1.147 \\
dev.\% & $0.68 \%$ & $0.74 \%$ & $0.8 \%$ & $0.87 \%$ & $0.95 \%$ & $1.04 \%$ & $1.34 \%$ & $1.22 \%$ & $1.27 \%$ \\
\hline$q=8$ & & & & & & & & & \\
init & 182.026 & 167.286 & 154.511 & 143.338 & 132.977 & 121.883 & 111.371 & 103.086 & 97.368 \\
unif & 180.879 & 166.140 & 153.364 & 142.191 & 131.831 & 120.737 & 110.225 & 101.940 & 96.221 \\
dev & 1.147 & 1.146 & 1.147 & 1.147 & 1.146 & 1.146 & 1.146 & 1.146 & 1.147 \\
dev.\% & $0.63 \%$ & $0.69 \%$ & $0.74 \%$ & $0.80 \%$ & $0.86 \%$ & $0.94 \%$ & $1.03 \%$ & $1.11 \%$ & $1.18 \%$ \\
\hline$q=9$ & & & & & & & & & \\
init & 196.613 & 180.907 & 167.232 & 155.399 & 144.928 & 134.479 & 123.167 & 113.331 & 105.865 \\
unif & 195.466 & 179.760 & 166.086 & 154.252 & 143.781 & 133.333 & 122.020 & 112.185 & 104.717 \\
dev & 1.147 & 1.147 & 1.146 & 1.147 & 1.147 & 1.146 & 1.147 & 1.146 & 1.148 \\
dev.\% & $0.58 \%$ & $0.63 \%$ & $0.69 \%$ & $0.74 \%$ & $0.79 \%$ & $0.85 \%$ & $0.93 \%$ & $1.01 \%$ & $1.08 \%$ \\
\hline
\end{tabular}

From the above table, the succeeding outcomes are summarized as follows:

(1) The total costs calculated from initial optimality Equation (5) are nearly approximated to the outcomes acquired by modified optimality equation of uniformization (8) with a tiny bias less than 1.147 .

(2) The outcomes demonstrate that the total discount cost function $\mathrm{U}_{\alpha}(q, m)$ obtained Equations (5) and (8) are all increasing in the amount of customers, which has been demonstrated theoretically in Theorem 1.

(3) The outcomes demonstrate that the total discount cost function $U_{\alpha}(q, m)$ acquired from Equations (5) and (8) are all decreasing in the amount of working servers.

Therefore, it can be concluded that there exist similar properties and outcomes of the total discounted cost acquired from (5) and (8), respectively.

Afterwards, several examples are conducted to demonstrate the associated characteristics of cost difference function $\Delta\left(q_{q n}, m_{q n}\right)=T U_{\alpha}^{1}\left(q_{q n}, m_{q n}\right)-T U_{\alpha}^{2}\left(q_{q n}, m_{q n}\right)$ and find the corresponding thresholds to trigger group replacements based on different operating servers or specific amount of customers in system. The outcomes are presented in the following Table 2.

Table 2. Cost difference function $\Delta\left(q_{q n}, m_{q n}\right)$ and thresholds to trigger group replacements $M=8$, $\sigma_{m}=5, m=0, \ldots, 8, \zeta=3, \beta=1, F=25, r_{c}=2, k=20, \gamma=4, j=4, \alpha=0.8$, iteration $=300$.

\begin{tabular}{cccccccccc}
\hline $\boldsymbol{m}$ & $\mathbf{0}$ & $\mathbf{1}$ & $\mathbf{2}$ & $\mathbf{3}$ & $\mathbf{4}$ & $\mathbf{5}$ & $\mathbf{6}$ & $\mathbf{7}$ & $\mathbf{8}$ \\
\hline$q=0$ & & & & & & & & & \\
act1 & 81.696 & 71.998 & 65.288 & 60.428 & 56.778 & 53.954 & 51.712 & 49.895 & 48.393 \\
act2 & 81.982 & 72.652 & 66.285 & 61.794 & 58.608 & 56.476 & 55.531 & 57.478 & - \\
dif & -0.286 & -0.654 & -0.997 & -1.366 & -1.83 & -2.522 & -3.819 & -7.583 & - \\
unif & 81.696 & 71.998 & 65.288 & 60.428 & 56.778 & 53.954 & 51.712 & 49.895 & 48.393 \\
\hline
\end{tabular}


Table 2. Cont

\begin{tabular}{|c|c|c|c|c|c|c|c|c|c|}
\hline$m$ & 0 & 1 & 2 & 3 & 4 & 5 & 6 & 7 & 8 \\
\hline \multicolumn{10}{|l|}{$q=1$} \\
\hline act1 & 94.952 & 81.761 & 73.233 & 67.363 & 63.126 & 59.946 & 57.480 & 55.517 & 53.918 \\
\hline act2 & 94.951 & 82.235 & 74.110 & 68.645 & 64.895 & 62.421 & 61.263 & 63.071 & - \\
\hline dif & 0.001 & -0.474 & -0.877 & -1.282 & -1.769 & -2.475 & -3.783 & -7.554 & - \\
\hline unif & 94.951 & 81.761 & 73.233 & 67.363 & 63.126 & 59.946 & 57.480 & 55.517 & 53.918 \\
\hline \multicolumn{10}{|l|}{$q=3$} \\
\hline act1 & 118.316 & 106.685 & 94.277 & 83.746 & 77.121 & 72.636 & 69.418 & 66.998 & 65.112 \\
\hline act2 & 117.878 & 106.580 & 94.666 & 84.720 & 78.682 & 74.963 & 73.090 & 74.467 & - \\
\hline dif & 0.438 & 0.105 & -0.389 & -0.974 & -1.561 & -2.327 & -3.672 & -7.469 & - \\
\hline unif & 117.878 & 106.580 & 94.277 & 83.746 & 77.121 & 72.636 & 69.418 & 66.998 & 65.112 \\
\hline \multicolumn{10}{|l|}{$q=5$} \\
\hline act1 & 142.568 & 130.123 & 118.738 & 106.933 & 95.886 & 87.625 & 82.530 & 79.113 & 76.663 \\
\hline act2 & 141.675 & 129.534 & 118.498 & 107.214 & 96.910 & 89.611 & 85.970 & 86.414 & - \\
\hline dif & 0.893 & 0.589 & 0.240 & -0.281 & -1.024 & -1.986 & -3.440 & -7.301 & - \\
\hline unif & 141.675 & 129.534 & 118.498 & 106.933 & 95.886 & 87.625 & 82.530 & 79.113 & 76.663 \\
\hline \multicolumn{10}{|l|}{$q=6$} \\
\hline act1 & 155.262 & 142.054 & 130.385 & 119.175 & 107.573 & 97.469 & 90.167 & 85.698 & 82.711 \\
\hline act2 & 154.131 & 141.225 & 129.881 & 119.093 & 108.178 & 99.079 & 93.370 & 92.840 & - \\
\hline dif & 1.131 & 0.829 & 0.504 & 0.082 & -0.605 & -1.610 & -3.203 & -7.142 & - \\
\hline unif & 154.131 & 141.225 & 129.881 & 119.093 & 107.573 & 97.469 & 90.167 & 85.698 & 82.711 \\
\hline \multicolumn{10}{|l|}{$q=8$} \\
\hline act1 & 182.502 & 167.453 & 154.362 & 142.844 & 132.025 & 120.737 & 110.225 & 101.94 & 96.221 \\
\hline act2 & 180.879 & 166.140 & 153.364 & 142.191 & 131.831 & 121.380 & 112.455 & 108.269 & - \\
\hline dif & 1.623 & 1.313 & 0.998 & 0.653 & 0.194 & -0.643 & -2.230 & -6.329 & - \\
\hline unif & 180.879 & 166.140 & 153.364 & 142.191 & 131.831 & 120.737 & 110.225 & 101.940 & 96.221 \\
\hline \multicolumn{10}{|l|}{$q=10$} \\
\hline act1 & 212.880 & 195.942 & 181.030 & 168.070 & 156.765 & 146.256 & 134.617 & 123.577 & 114.500 \\
\hline act2 & 210.823 & 194.213 & 179.635 & 167.027 & 156.124 & 146.187 & 135.938 & 128.907 & - \\
\hline dif & 2.057 & 1.729 & 1.395 & 1.043 & 0.641 & 0.069 & -1.321 & -5.330 & - \\
\hline unif & 210.823 & 194.213 & 179.635 & 167.027 & 156.124 & 146.187 & 134.617 & 123.577 & 114.500 \\
\hline \multicolumn{10}{|l|}{$q=18$} \\
\hline act1 & 356.441 & 334.560 & 314.346 & 295.996 & 279.741 & 265.875 & 254.628 & 240.777 & 225.093 \\
\hline act2 & 353.203 & 331.688 & 311.878 & 293.980 & 278.243 & 264.993 & 254.558 & 244.142 & - \\
\hline dif & 3.238 & 2.872 & 2.468 & 2.016 & 1.498 & 0.882 & 0.069 & -3.366 & - \\
\hline unif & 353.203 & 331.688 & 311.878 & 293.980 & 278.243 & 264.993 & 254.558 & 240.777 & 225.093 \\
\hline \multicolumn{10}{|l|}{$q=500$} \\
\hline act1 & $12,187.7$ & $12,158.3$ & $12,130.2$ & $12,103.6$ & $12,079.0$ & $12,056.9$ & $12,038.5$ & 12,022 & $12,001.3$ \\
\hline act2 & $12,182.5$ & $12,153.5$ & $12,125.9$ & $12,099.8$ & $12,075.9$ & $12,054.7$ & $12,037.4$ & $12,023.8$ & - \\
\hline dif & 5.2 & 4.8 & 4.3 & 3.8 & 3.1 & 2.2 & 1.1 & -1.8 & - \\
\hline unif & $12,182.5$ & $12,153.5$ & $12,125.9$ & $12,099.8$ & $12,075.9$ & $12,054.7$ & $12,037.4$ & 12,022 & $12,001.3$ \\
\hline \multicolumn{10}{|l|}{$q=7000$} \\
\hline act1 & $172,872.5$ & 172,843 & 172,815 & 172,788 & 172,764 & 172,742 & 172,723 & 172,707 & 172,686 \\
\hline act2 & $172,867.3$ & $172,838.3$ & $172,810.6$ & 172,785 & 172,761 & 172,740 & 172,722 & $172,708.8$ & - \\
\hline dif & 5.2 & 4.7 & 4.4 & 3 & 3 & 2 & 1 & -1.8 & - \\
\hline unif & $172,867.3$ & $172,838.3$ & $172,810.6$ & 172,785 & 172,761 & 172,740 & 172,722 & 172,707 & 172,686 \\
\hline
\end{tabular}

From the above table, the following consequences can be summarized as:

(1) The cost difference function of $T U_{\alpha}^{1}\left(q_{q n}, m_{q n}\right)-T U_{\alpha}^{2}\left(q_{q n}, m_{q n}\right)$ is increasing in the amount of customers for every specific amount of working servers, which has been shown previously in Theorem 3.

(2) In the light of the above consequence of (1), numerical results of Table 2, and Theorem 4, the amount of customers to activate the group replacement while $m_{q n}$ servers are normally working, $q_{q n}^{*}\left(m_{q n}\right)$, is presented as follows:

$q_{q n}^{*}(0)=1$ : In the situation of all servers are broken down, the group replacement is activated 
while the amount of customers raises to 1 .

$q_{q n}^{*}(1)=3$ : When one servers is normally working, the group replacement is triggered while the amount of customers increases to 3 .

Moreover, $q_{q n}^{*}(2)=5, q_{q n}^{*}(3)=6, q_{q n}^{*}(4)=8, q_{q n}^{*}(5)=10, q_{q n}^{*}(6)=18, q_{q n}^{*}(7)=\infty, q_{q n}^{*}(8)=\infty$. Consequently, according to above numerical results, it can be concluded that the group replacement will never be conducted no matter how high the amount of customers in the system after the amount of working servers reaches to 7 .

(3) Table 2 also demonstrates that the cost difference function of $T U_{\alpha}^{1}\left(q_{q n}, m_{q n}\right)-T U_{\alpha}^{2}\left(q_{q n}, m_{q n}\right)$ is decreasing in the number of normally working servers for each respective amount of customers in the system.

(4) According the above numerical results of Table 2, the threshold of the number of working servers to activate the group replacement with $q_{q n}$ customers in the system, $m_{q n}^{*}\left(q_{q n}\right)$, is presented as:

$$
m_{q n}^{*}(0)=\text { none, } m_{q n}^{*}(1)=\text { none, } m_{q n}^{*}(2)=\text { none. }
$$

When there are from 0 to 2 customers in the system, the group replacement will not be activated even all servers are failed.

$$
m_{q n}^{*}(3)=1 \text {. }
$$

When there are 3 customers in the system, the group replacement is activated when the amount of operating servers is reduced to 1 .

Moreover, $m_{q n}^{*}(4)=1, m_{q n}^{*}(5)=2, m_{q n}^{*}(6)=3, m_{q n}^{*}(7)=3, m_{q n}^{*}(8)=4, m_{q n}^{*}(9)=4, m_{q n}^{*}(10)=5$, $m_{q n}^{*}(11)=5, m_{q n}^{*}(12)=5, m_{q n}^{*}(13)=5, m_{q n}^{*}(14)=5, m_{q n}^{*}(15)=5, m_{q n}^{*}(16)=5, m_{q n}^{*}(17)=5$.

In these cases of 10 to 17 customers in the system, the group replacements are triggered while the amount of operating servers is dropped to 5 .

$$
\begin{aligned}
& m_{q n}^{*}(18)=6, m_{q n}^{*}(19)=6, m_{q n}^{*}(20)=6, m_{q n}^{*}(30)=6, m_{q n}^{*}(40)=6, m_{q n}^{*}(50)=6, m_{q n}^{*}(100)=6, m_{q n}^{*}(200)=6, \\
& m_{q n}^{*}(300)=6, m_{q n}^{*}(400)=6, m_{q n}^{*}(500)=6, m_{q n}^{*}(1000)=6, m_{q n}^{*}(2000)=6, m_{q n}^{*}(3000)=6, m_{q n}^{*}(4000)=6, \\
& m_{q n}^{*}(5000)=6, m_{q n}^{*}(6000)=6, m_{q n}^{*}(7000)=6 .
\end{aligned}
$$

In these cases of from 18 to 7000 customers in the system, the group replacements are started while the amount of operating servers is decreased to 6 .

Actually, according to our numerical results, the cost difference function of $T U_{\alpha}^{1}\left(q_{q n}, m_{q n}\right)-$ $T U_{\alpha}^{2}\left(q_{q n}, m_{q n}\right)$ with 7 operating servers converges to around -1.874 after the amount of customers in system reaches to 100 , consequently the threshold of the amount of normally operating servers to start the group replacement is 6 while there are more than 17 customers in the system.

\section{Conclusions}

This proposed IoT conditioned-based group replacement decision support system first develops the discounted cost model for a service/production system with numerous independent working servers. The original discounted cost model is further revised into an equivalent model to facilitate the proof procedure by applying the uniformization approach. Several significant theoretical properties are proven and many numerical examples are conducted for two kinds of group replacement policies, respectively.

For the first kind of group replacement policy, Theorem 1, Lemma 2, Theorems 2-4 certainly prove that there is a threshold of the amount of customers necessary to activate the group replacement depending on various amount of working servers. These theorems are very difficult to be certified and are the most significant theoretical breakthroughs and the main contribution in this paper. Moreover, numerical examples conducted in Table 2 can also illustrate the above theoretical outcomes already derived for the first class of group replacement policy. Besides, for the second class of group replacement 
policy, the numerical results of Table 2 definitely confirm that there is a threshold of amount of working servers existed to start the group replacement according to distinct amount of customers in the system.

According to the perspective view of management, our proposed decision support system applies a computer monitoring system to instantaneously evaluate the status of servers by collecting the precise operating data from desired IoT sensors, and the status of customers in the system by collecting the signals of built-in IoT devices from these customers, and further decides whether the group replacement should be conducted based on the group replacement threshold.

Consequently, based on the structure and detailed procedure flow presented in Figures 1 and 2, this proposed IoT condition-based group replacement decision support system can be used to solve the replacement problems of some designated service or production systems with several machines or servers operating in parallel, such as telecommunication and customized production systems, internet service systems, or some parallel subsystems deployed in submarines, satellites, and space stations.

Funding: This research received no external funding.

Acknowledgments: The author sincerely appreciate the valuable comments and suggestions given by reviewers and Assistant Editor of Mathematics.

Conflicts of Interest: The author declares no conflict of interest.

\section{Appendix A}

\section{Proof of Theorem 1.}

The proof will be conducted by induction on $n$.

For $n=1$, we have

$$
T U_{\alpha}\left(q_{q n}, m_{q n} ; n=1\right)=\min \left\{\frac{k q_{q n}}{\bar{\sigma}}, \frac{\frac{j \gamma}{M_{q n}-m_{q n}}}{\bar{\sigma}}\left[F+\left(M_{q n}-m_{q n}\right) r_{c}\right]+\frac{k q_{q n}}{\bar{\sigma}}\right\}
$$

where $\bar{\sigma} \geq \alpha+\sigma+M_{q n} \zeta+M_{q n} \beta+j \gamma$.

$T U_{\alpha}\left(q_{q n}, m_{q n} ; n=1\right)$ is the minimum of two increasing functions of $q_{q n}$, therefore it is obviously increasing in $q_{q n}$. Suppose $T U_{\alpha}\left(q_{q n}, m_{q n} ; n\right)$ is increasing in $q_{q n}$, then now we need to prove $T U_{\alpha}\left(q_{q n}, m_{q n} ; n+1\right)$ is also increasing in $q_{q n}$.

As we know, $T U_{\alpha}\left(q_{q n}, m_{q n} ; n+1\right)=\min \left\{T U_{\alpha}^{1}\left(q_{q n}, m_{q n} ; n+1\right), T U_{\alpha}^{2}\left(q_{q n}, m_{q n} ; n+1\right)\right\}$, and if both of $T U_{\alpha}^{1}\left(q_{q n}, m_{q n} ; n+1\right)$ and $T U_{\alpha}^{2}\left(q_{q n}, m_{q n} ; n+1\right)$ are increasing in $q_{q n}$, then the result holds. We prove these two properties next.

(1) $\operatorname{TU}_{\alpha}^{1}\left(q_{q n}, m_{q n} ; n+1\right)$ is increasing in $q_{q n}$.

Recall (9), it needs to be showed that $T U^{1}\left(q_{q n}, m_{q n} ; n+1\right)-T U^{1}\left(q_{q n}+1, m_{q n} ; n+1\right) \leq 0$, for all $m_{q n}$. To show the inequality, two cases for $q_{q n}$ are considered:

Case 1. When $q_{q n}<m_{q n}$ :

Then $\min \left(q_{q n}, m_{q n}\right)=q_{q n}, \min \left(q_{q n}+1, m_{q n}\right)=q_{q n}+1$, and

$$
\begin{aligned}
& T U^{1}\left(q_{q n}, m_{q n} ; n+1\right)-T U^{1}\left(q_{q n}+1, m_{q n} ; n+1\right) \\
& =-\frac{k}{\bar{\sigma}}+\frac{\sigma}{\bar{\sigma}}\left[T U_{\alpha}\left(q_{q n}+1, m_{q n} ; n\right)-T U_{\alpha}\left(q_{q n}+2, m_{q n} ; n\right)\right]+\frac{m_{q n} \beta}{\bar{\sigma}}\left[T U_{\alpha}\left(q_{q n}, m_{q n}-1 ; n\right)-\right. \\
& \left.T U_{\alpha}\left(q_{q n}+1, m_{q n}-1 ; n\right)\right]+\frac{q_{q n} \zeta}{\bar{\sigma}}\left[T U_{\alpha}\left(q_{q n}-1, m_{q n} ; n\right)-T U_{\alpha}\left(q_{q n}, m_{q n} ; n\right)\right]-\frac{\zeta}{\bar{\sigma}} T U_{\alpha}\left(q_{q n}, m_{q n} ; n\right) \\
& +\left(1-\frac{\alpha+\sigma+m_{q n} \beta+\left(q_{q n}+1\right) \zeta}{\bar{\sigma}}\right)\left[T U_{\alpha}\left(q_{q n}, m_{q n} ; n\right)-T U_{\alpha}\left(q_{q n}+1, m_{q n} ; n\right)\right]+\frac{\zeta}{\bar{\sigma}} T U_{\alpha}\left(q_{q n}, m_{q n} ; n\right) \\
& =-\frac{k}{\bar{\sigma}}+\frac{\sigma}{\bar{\sigma}}\left[T U_{\alpha}\left(q_{q n}+1, m_{q n} ; n\right)-T U_{\alpha}\left(q_{q n}+2, m_{q n} ; n\right)\right]+\frac{m_{q n} \beta}{\bar{\sigma}}\left[T U_{\alpha}\left(q_{q n}, m_{q n}-1 ; n\right)-\right. \\
& \left.T U_{\alpha}\left(q_{q n}+1, m_{q n}-1 ; n\right)\right]+\frac{q_{q n} \zeta}{\bar{\sigma}}\left[T U_{\alpha}\left(q_{q n}-1, m_{q n} ; n\right)-T U_{\alpha}\left(q_{q n}, m_{q n} ; n\right)\right] \\
& +\left(1-\frac{\alpha+\sigma+m_{q n} \beta+\left(q_{q n}+1\right) \zeta}{\bar{\sigma}}\right)\left[T U_{\alpha}\left(q_{q n}, m_{q n} ; n\right)-T U_{\alpha}\left(q_{q n}+1, m_{q n} ; n\right)\right] .
\end{aligned}
$$


Because all items in brackets are non-positive, $T U^{1}\left(q_{q n}, m_{q n} ; n+1\right)-T U^{1}\left(q_{q n}+1, m_{q n} ; n+1\right) \leq 0$ for $q_{q n}<m_{q n}$.

Case 2. When $q_{q n} \geq m_{q n}$ :

Then $q_{q n}+1>m_{q n}$ and $\min \left(q_{q n}, m_{q n}\right)=\min \left(q_{q n}+1, m_{q n}\right)=m_{q n}$, and the difference $T U^{1}\left(q_{q n}, m_{q n} ; n+1\right)-T U^{1}\left(q_{q n}+1, m_{q n} ; n+1\right)$, according to the induction hypothesis, is non-positive due to all items in brackets are non-positive.

Now since $T U^{1}\left(q_{q n}, m_{q n} ; n+1\right)-T U^{1}\left(q_{q n}+1, m_{q n} ; n+1\right) \leq 0$ for all $q_{q n}$, it can be concluded that $T U^{1}\left(q_{q n}, m_{q n} ; n+1\right)$ is increasing in $q_{q n}$.

(2) $T U_{\alpha}^{2}\left(q_{q n}, m_{q n} ; n+1\right)$ is increasing in $q_{q n}$.

Recall (9), it needs to show that $T U^{2}\left(q_{q n}, m_{q n} ; n+1\right)-T U^{2}\left(q_{q n}+1, m_{q n} ; n+1\right) \leq 0$. Two cases for $q_{q n}$ are considered.

Case 1. $q_{q n}<m_{q n}$ :

It is clear that $\min \left(q_{q n}, m_{q n}\right)=q_{q n}, \min \left(q_{q n}+1, m_{q n}\right)=q_{q n}+1$, and

$$
\begin{aligned}
& T U^{2}\left(q_{q n}, m_{q n} ; n+1\right)-T U^{2}\left(q_{q n}+1, m_{q n} ; n+1\right) \\
& =-\frac{k}{\bar{\sigma}}+\frac{\sigma}{\bar{\sigma}}\left[T U_{\alpha}\left(q_{q n}+1, m_{q n} ; n\right)-T U_{\alpha}\left(q_{q n}+2, m_{q n} ; n\right)\right]+\frac{m_{q n} \beta}{\bar{\sigma}}\left[T U_{\alpha}\left(q_{q n}, m_{q n}-1 ; n\right)-\right. \\
& \left.T U_{\alpha}\left(q_{q n}+1, m_{q n}-1 ; n\right)\right]+\frac{q_{q n} \zeta}{\bar{\sigma}}\left[T U_{\alpha}\left(q_{q n}-1, m_{q n} ; n\right)-T U_{\alpha}\left(q_{q n}, m_{q n} ; n\right)\right]-\frac{\zeta}{\bar{\sigma}} T U_{\alpha}\left(q_{q n}, m_{q n} ; n\right)+ \\
& \frac{\frac{j \eta}{M_{q q n}-m_{q n}}}{\bar{\sigma}}\left[T U_{\alpha}\left(q_{q n}, M_{q n} ; n\right)-T U_{\alpha}\left(q_{q n}+1, M_{q n} ; n\right)\right]+\left(1-\frac{\alpha+\sigma+m \beta+q q n \zeta+\frac{i \nu}{M_{q n}-m_{q n}}}{\bar{\sigma}}\right) * \\
& {\left[T U_{\alpha}\left(q_{q n}, m_{q n} ; n\right)-T U_{\alpha}\left(q_{q n}+1, m_{q n} ; n\right)\right]+\frac{\zeta}{\bar{\sigma}} T U_{\alpha}\left(q_{q n}+1, m_{q n} ; n\right)} \\
& =-\frac{k}{\bar{\sigma}}+\frac{\sigma}{\bar{\sigma}}\left[T U_{\alpha}\left(q_{q n}+1, m_{q n} ; n\right)-T U_{\alpha}\left(q_{q n}+2, m_{q n} ; n\right)\right]+\frac{m_{q n} \beta}{\bar{\sigma}}\left[T U_{\alpha}\left(q_{a n}, m_{q n}-1 ; n\right)-T U_{\alpha}\left(q_{q n}+1, m_{q n}-1 ; n\right)\right] \\
& +\frac{q_{q n} \zeta}{\bar{\sigma}}\left[T U_{\alpha}\left(q_{q n}-1, m_{q n} ; n\right)-T U_{\alpha}\left(q_{q n}, m_{q n} ; n\right)\right]+\frac{\frac{j \eta}{M_{q n}-m_{q n}}}{\bar{\sigma}}\left[T U_{\alpha}\left(q_{q n}, M_{q n} ; n\right)-T U_{\alpha}\left(q_{q n}+1, M_{q n} ; n\right)\right] \\
& +\left(1-\frac{\alpha+\sigma+m \beta+(q+1) \zeta+\frac{j \gamma}{M q n-m q n}}{\bar{\sigma}}\right)\left[T U_{\alpha}\left(q_{q n}, m_{q n} ; n\right)-T U_{\alpha}\left(q_{q n}+1, m_{q n} ; n\right)\right] \leq 0 .
\end{aligned}
$$

Since all items in the above brackets are not positive based on the induction hypothesis, $T U^{2}\left(q_{q n}, m_{q n} ; n+1\right)-T U^{2}\left(q_{q n}+1, m_{q n} ; n+1\right) \leq 0$ for $q_{q n}<m_{q n}$.

Case 2. $q_{q n} \geq m_{q n}$ :

It is obvious that $q_{q n}+1>m_{q n}$ and $\min \left(q_{q n}, m_{q n}\right)=\min \left(q_{q n}+1, m_{q n}\right)=m_{q n}$, and the difference $T U^{2}\left(q_{q n}, m_{q n} ; n+1\right)-T U^{2}\left(q_{q n}+1, m_{q n} ; n+1\right)$ for $q_{q n} \geq m_{q n}$, according to the induction hypothesis, is non-positive due to all items in brackets are non-positive.

Since $T U^{2}\left(q_{q n}, m_{q n} ; n+1\right)-T U^{2}\left(q_{q n}+1, m_{q n} ; n+1\right) \leq 0$ for all $q_{q n}$, it can be concluded that $T U^{2}\left(q_{q n}, m_{q n} ; n+1\right)$ is increasing in $q_{q n}$.

(3) $\mathrm{TU}_{\alpha}\left(q_{q n}, m_{q n} ; n+1\right)$ is the minimum of two non-decreasing functions, and this judges that $T U_{\alpha}\left(q_{q n}, m_{q n} ; n+1\right)$ is also non-decreasing in $q_{q n}$ and completes the induction proof. Consequently, $T U_{\alpha}\left(q_{q n}, m_{q n} ; n\right)$ is increasing in $q_{q n}$ for all $\mathrm{n}$, and by applying standard contentions from successive approximation for discounted Markov decision process, $T U_{\alpha}\left(q_{q n}, m_{q n}\right)=\lim _{n} T U_{\alpha}\left(q_{q n}, m_{q n} ; n\right)$ is also increasing in $q_{q n}$. The proof is done.

\section{Proof of Theorem 2.}

Since $T U_{\alpha}\left(q_{q n}, m_{q n}\right)=\min \left\{T U_{\alpha}^{1}\left(q_{q n}, m_{q n}\right), T U_{\alpha}^{2}\left(q_{q n}, m_{q n}\right)\right\}$, two cases are considered as follows: (1) $T U_{\alpha}\left(q_{q n}, m_{q n}\right)=T U_{\alpha}^{2}\left(q_{q n}, m_{q n}\right)$ :

The proof is conducted by induction on $n$. From (9), for $n=1$, we have

$$
\begin{aligned}
& T U_{\alpha}\left(q_{q n}, m_{q n} ; n=1\right)-T U_{\alpha}\left(q_{q n}, M_{q n} ; n=1\right)=T U_{\alpha}^{2}\left(q_{q n}, m_{q n} ; n=1\right)-T U_{\alpha}^{1}\left(q_{q n}, M_{q n} ; n=1\right) \\
& =\frac{\frac{i \gamma}{M q n-m q n}}{\bar{\sigma}}+\frac{k q q n}{\bar{\sigma}}-\frac{k q_{q n}}{\bar{\sigma}}=\frac{\frac{i \gamma}{M q n-m q n}}{\bar{\sigma}} \text {. }
\end{aligned}
$$

It is obvious that $T U_{\alpha}\left(q_{q n}, m_{q n} ; n=1\right)-T U_{\alpha}\left(q_{q n}, M_{q n} ; n=1\right)$ is non-decreasing in $q_{q n}$. 
Assume that $T U_{\alpha}\left(q_{q n}, m_{q n} ; n\right)-T U_{\alpha}\left(q_{q n}, M_{q n} ; n\right)$ is increasing in $q_{q n}$, then it must be shown that $T U_{\alpha}\left(q_{q n}, m_{q n} ; n+1\right)-T U_{\alpha}\left(q_{q n}, M_{q n} ; n+1\right)$ is also increasing in $q_{q n}$. From (9), it can be seen that

$$
\begin{aligned}
& T U_{\alpha}\left(q_{q n}, m_{q n} ; n+1\right)-T U_{\alpha}\left(q_{q n}, M_{q n} ; n+1\right)=T U_{\alpha}^{2}\left(q_{q n}, m_{q n} ; n+1\right)-T U_{\alpha}^{1}\left(q_{q n}, M_{q n} ; n+1\right) \\
& =\frac{\overline{j q n}}{\overline{M_{q n}-m_{q n}}}\left[F+\left(M_{q n}-m_{q n}\right) r_{c}\right]+\frac{\sigma}{\bar{\sigma}}\left[T U_{\alpha}\left(q_{q n}+1, m_{q n} ; n\right)-T U_{\alpha}\left(q_{q n}+1, M_{q n} ; n\right)\right]+ \\
& {\left[\frac { \zeta \operatorname { m i n } ( q _ { q n } , m _ { q n } ) } { \overline { \sigma } } \left[T U_{\alpha}\left(q_{q n}-1, m_{q n} ; n\right)-\frac{\zeta \min \left(q_{q n}, M_{q n}\right)}{\bar{\sigma}} T U_{\alpha}\left(q_{q n}-1, M_{q n} ; n\right)+\right.\right.} \\
& {\left[\frac{m_{q n} \beta}{\bar{\sigma}} T U_{\alpha}\left(q_{q n}, m_{q n}-1 ; n\right)-\frac{M_{q n} \beta}{\bar{\sigma}} T U_{\alpha}\left(q_{q n}, M_{q n}-1 ; n\right)\right]+\frac{\overline{M_{q n}-m_{q n}}}{\bar{\sigma}} T U_{\alpha}\left(q_{q n}, M_{q n} ; n\right)+} \\
& {\left[\left(1-\frac{\alpha+\sigma\left(q_{q n}, m_{q n}\right)+\frac{j \gamma}{M_{q n}-m_{q n}}}{\bar{\sigma}}\right) T U_{\alpha}\left(q_{q n}, m_{q n} ; n\right)-\left(1-\frac{\alpha+\sigma\left(q_{q n}, M_{q n}\right)}{\bar{\sigma}}\right) T U_{\alpha}\left(q_{q n}, M_{q n} ; n\right)\right] .}
\end{aligned}
$$

Now we consider three cases for $q_{q n}$ :

Case 1. $q_{q n} \geq M_{q n}$

For this case, $\min \left(q_{q n}, m_{q n}\right)=m_{q n}$ and $\min \left(q_{q n}, M_{q n}\right)=M_{q n}$, hence (A1) becomes

$T U_{\alpha}\left(q_{q n}, m_{q n} ; n+1\right)-T U_{\alpha}\left(q_{q n}, M_{q n} ; n+1\right)$

$=\frac{\frac{j \gamma}{M_{q n}-m_{q n}}}{\bar{\sigma}}\left[F+\left(M_{q n}-m_{q n}\right) r_{c}\right]+\frac{\sigma}{\bar{\sigma}}\left[T U_{\alpha}\left(q_{q n}+1, m_{q n} ; n\right)-T U_{\alpha}\left(q_{q n}+1, M_{q n} ; n\right)\right]+$

$\frac{m_{q n} \zeta}{\bar{\sigma}}\left[T U_{\alpha}\left(q_{q n}-1, m_{q n} ; n\right)-T U_{\alpha}\left(q_{q n}-1, M_{q n} ; n\right)\right]-\frac{\left(M_{q n}-m_{q n}\right) \zeta}{\bar{\sigma}} T U_{\alpha}\left(q_{q n}-1, M_{q n} ; n\right)+$

$\frac{m_{q n} \beta}{\bar{\sigma}}\left[T U_{\alpha}\left(q_{q n}, m_{q n}-1 ; n\right)-T U_{\alpha}\left(q_{q n}, M_{q n}-1 ; n\right)\right]-\frac{\left(M_{q n}-m_{q n}\right) \beta}{\bar{\sigma}} T U_{\alpha}\left(q_{q n}, M_{q n}-1 ; n\right)+$

$\frac{\frac{j \gamma}{M q n-m q n}}{\bar{\sigma}} T U_{\alpha}\left(q_{q n}, M_{q n} ; n\right)+\left(1-\frac{\alpha+\sigma+M_{q n} \zeta+M_{q n} \beta+\frac{j \gamma}{M q n-m q n}}{\bar{\sigma}}\right)\left[T U_{\alpha}\left(q_{q n}, m_{q n} ; n\right)-\right.$

$\left.T U_{\alpha}\left(q_{q n}, M_{q n} ; n\right)\right]+\frac{\left(M_{q n}-m_{q n}\right) \zeta}{\bar{\sigma}} T U_{\alpha}\left(q_{q n}, m_{q n} ; n\right)+\frac{\left(M_{q n}-m_{q n}\right) \beta}{\bar{\sigma}} T U_{\alpha}\left(q_{q n}, m_{q n} ; n\right)-$

$\frac{\frac{j \gamma}{M q n-m q n}}{\bar{\sigma}} T U_{\alpha}\left(q_{q n}, M_{q n} ; n\right)$

$=\frac{\frac{j \gamma}{M_{q n}-m_{q n}}}{\bar{\sigma}}\left[F+\left(M_{q n}-m_{q n}\right) r_{c}\right]+\frac{\sigma}{\bar{\sigma}}\left[T U_{\alpha}\left(q_{q n}+1, m_{q n} ; n\right)-T U_{\alpha}\left(q_{q n}+1, M_{q n} ; n\right)\right]+$

$+\frac{m_{q n} \zeta}{\bar{\sigma}}\left[T U_{\alpha}\left(q_{q n}-1, m_{q n} ; n\right)-T U_{\alpha}\left(q_{q n}-1, M_{q n} ; n\right)\right]+\frac{\left(M_{q n}-m_{q n}\right) \zeta}{\bar{\sigma}}\left[T U_{\alpha}\left(q_{q n}, m_{q n} ; n\right)-T U_{\alpha}\left(q_{q n}-1, M_{q n} ; n\right)\right]$

$+\frac{m_{q n} \beta}{\bar{\sigma}}\left[T U_{\alpha}\left(q_{q n}, m_{q n}-1 ; n\right)-T U_{\alpha}\left(q_{q n}, M_{q n}-1 ; n\right)\right]+\frac{\left(M_{q n}-m_{q n}\right) \beta}{\bar{\sigma}}\left[T U_{\alpha}\left(q_{q n}, m_{q n} ; n\right)-T U_{\alpha}\left(q_{q n}, M_{q n}-1 ; n\right)\right]$

$+\left(1-\frac{\alpha+\sigma+M_{q n} \zeta+M_{q n} \beta+\frac{j \gamma}{M_{q n}-m_{q n}}}{\bar{\sigma}}\right)\left[T U_{\alpha}\left(q_{q n}, m_{q n} ; n\right)-T U_{\alpha}\left(q_{q n}, M_{q n} ; n\right)\right]$.

After rearranging terms, we obtain

$$
\begin{aligned}
& T U_{\alpha}\left(q_{q n}, m_{q n} ; n+1\right)-T U_{\alpha}\left(q_{q n}, M_{q n} ; n+1\right) \\
& =\frac{\frac{j \gamma}{M q n-m q n}}{\bar{\sigma}}\left[F+\left(\ddot{M}_{q n}-m_{q n}\right) r_{c}\right]+\frac{\sigma}{\bar{\sigma}}\left[T U_{\alpha}\left(q_{q n}+1, m_{q n} ; n\right)-T U_{\alpha}\left(q_{q n}+1, M_{q n} ; n\right)\right]+ \\
& \frac{m_{q n} \zeta}{\bar{\sigma}}\left[T U_{\alpha}\left(q_{q n}-1, m_{q n} ; n\right)-T U_{\alpha}\left(q_{q n}-1, M_{q n} ; n\right)\right]+\frac{\left(M_{q n}-m_{q n}\right) \zeta}{\bar{\sigma}}\left[T U_{\alpha}\left(q_{q n}, m_{q n} ; n\right)-T U_{\alpha}\left(q_{q n}, M_{q n} ; n\right)\right] \\
& +\frac{m_{q n} \beta}{\bar{\sigma}}\left[T U_{\alpha}\left(q_{q n}, m_{q n}-1 ; n\right)-T U_{\alpha}\left(q_{q n}, M_{q n} ; n\right)\right]+\frac{\left(M_{q n}-m_{q n}\right) \beta}{\bar{\sigma}}\left[T U_{\alpha}\left(q_{q n}, m_{q n} ; n\right)-T U_{\alpha}\left(q_{q n}, M_{q n} ; n\right)\right] \\
& +\left\{\left(1-\frac{\alpha+\sigma+M_{q n} \zeta+M_{q n} \beta+\frac{j \gamma}{M_{q n}-m_{q n}}}{\bar{\sigma}}\right) *\left[T U_{\alpha}\left(q_{q n}, m_{q n} ; n\right)-T U_{\alpha}\left(q_{q n}, M_{q n} ; n\right)\right]-\frac{\left(M_{q n}-m_{q n}\right) \zeta}{\bar{\sigma}} *\right. \\
& \left.\left[T U_{\alpha}\left(q_{q n}-1, M_{q n} ; n\right)-T U_{\alpha}\left(q_{q n}, M_{q n} ; n\right)\right]-\frac{M_{q n} \beta}{\bar{\sigma}}\left[T U_{\alpha}\left(q_{q n}, M_{q n}-1 ; n\right)-T U_{\alpha}\left(q_{q n}, M_{q n} ; n\right)\right]\right\} .
\end{aligned}
$$

According to the induction hypothesis, the six items in (A2) are increasing in $q_{q n}$.

To complete this proof, it needs to be showed that

$$
\begin{aligned}
& \Delta^{1}\left(q_{q n}, m_{q n} ; n+1\right)=\left(1-\frac{\alpha+\sigma+M_{q n} \zeta+M_{q n} \beta+\frac{j \gamma}{M_{q n}-m_{q n}}}{\bar{\sigma}}\right)\left[T U_{\alpha}\left(q_{q n}, m_{q n} ; n\right)-T U_{\alpha}\left(q_{q n}, M_{q n} ; n\right)\right] \\
& -\frac{\left(M_{q n}-m_{q n}\right) \zeta}{\bar{\sigma}}\left[T U_{\alpha}\left(q_{q n}-1, M_{q n} ; n\right)-T U_{\alpha}\left(q_{q n}, M_{q n} ; n\right)\right]-\frac{M_{q n} \beta}{\bar{\sigma}}\left[T U_{\alpha}\left(q_{q n}, M_{q n}-1 ; n\right)-T U_{\alpha}\left(q_{q n}, M_{q n} ; n\right)\right]
\end{aligned}
$$

is also increasing in $q_{q n}$. 
Recall that parameter $\bar{\sigma}$ applied in the uniformization approach should be greater than $\bar{\sigma}=$ $\alpha+\sigma+M_{q n} \beta+M_{q n} \zeta+j \gamma$. Now we choose $\bar{\sigma} \geq \alpha+\sigma+M_{q n}^{*} \beta+M_{q n}^{*} \zeta+j \gamma$, where $M_{q n}^{*}$ is sufficiently large number. Then we rearrange $\Delta^{1}\left(q_{q n}, m_{q n} ; n+1\right)$ as follows:

$$
\begin{aligned}
& \Delta^{1}(q, m ; n+1)=\left(1-\frac{\alpha+\sigma+M_{q n}^{*} \zeta+M_{q n}^{*} \beta+\frac{j \gamma}{M_{q n}^{*}-m_{q n}}}{\bar{\sigma}}\right)\left[T U_{\alpha}\left(q_{q n}, m_{q n} ; n\right)-T U_{\alpha}\left(q_{q n}, M_{q n} ; n\right)\right] \\
& +\frac{\left(M_{q n}^{*}-M_{q n}\right) \zeta+\left(M_{q n}^{*}-M_{q n}\right) \beta}{\bar{\sigma}}\left[T U_{\alpha}\left(q_{q n}, m_{q n} ; n\right)-T U_{\alpha}\left(q_{q n}, M_{q n} ; n\right)\right]-\frac{\left(M_{q n}-m_{q n}\right) \zeta}{\bar{\sigma}} * \\
& {\left[T U_{\alpha}\left(q_{q n}-1, M_{q n} ; n\right)-T U_{\alpha}\left(q_{q n}, M_{q n} ; n\right)\right]-\frac{M_{q n} \beta}{\bar{\sigma}}\left[T U_{\alpha}\left(q_{q n}, M_{q n}-1 ; n\right)-T U_{\alpha}\left(q_{q n}, M_{q n} ; n\right)\right] .}
\end{aligned}
$$

In the above expression, as a result of large $M_{q n}^{*}$, the coefficient of the second increasing term $\frac{\left(M_{q n}^{*}-M_{q n}\right) \zeta+\left(M_{q n}^{*}-M_{q n}\right) \beta}{\bar{\sigma}}$ is almost round 1, as the coefficient of the last two decreasing terms is extremely small. According to the outcome of Lemma 2, we also know $T U_{\alpha}\left(q_{q n}, m_{q n} ; n\right)-T U_{\alpha}\left(q_{q n}+1, m_{q n} ; n\right)$ is bounded for every state $\left(q_{q n}, m_{q n}\right)$. Therefore, the entire expression of $\Delta^{1}(q, m ; n+1)$ should be increasing in $q_{q n}$ owing to sufficiently large $M_{q n}^{*}$.

Therefore, $T U_{\alpha}\left(q_{q n}, m_{q n} ; n+1\right)-T U_{\alpha}\left(q_{q n}, M_{q n} ; n+1\right)$ is increasing in $q$ when $q_{q n} \geq M_{q n}$.

Case 2. $m_{q n} \leq q_{q n}<M_{q n}$

In this case, $\min \left(q_{q n}, M_{q n}\right)=q_{q n}$ and $\min \left(q_{q n}, m_{q n}\right)=m_{q n}$, therefore (A2) becomes

$$
\begin{aligned}
& T U_{\alpha}\left(q_{q n}, m_{q n} ; n+1\right)-T U_{\alpha}\left(q_{q n}, M_{q n} ; n+1\right) \\
& =\frac{\frac{j \gamma}{M q n-m_{q n}}}{\bar{\sigma}}\left[F+\left(M_{q n}-m_{q n}\right) r_{c}\right]+\frac{\sigma}{\bar{\sigma}}\left[T U_{\alpha}\left(q_{q n}+1, m_{q n} ; n\right)-T U_{\alpha}\left(q_{q n}+1, M_{q n} ; n\right)\right]+\frac{m_{q n} \zeta}{\bar{\sigma}}\left[T U_{\alpha}\left(q_{q n}-1, m_{q n} ; n\right)\right. \\
& \left.-T U_{\alpha}\left(q_{q n}-1, M_{q n} ; n\right)\right]-\frac{\left(q_{q n}-m_{q n}\right) \zeta}{\bar{\sigma}} T U_{\alpha}\left(q_{q n}-1, M_{q n} ; n\right)+\frac{m_{q n} \beta}{\bar{\sigma}}\left[T U_{\alpha}\left(q_{q n}, m_{q n}-1 ; n\right)-T U_{\alpha}\left(q_{q n}, M_{q n}-1 ; n\right)\right]- \\
& \frac{\left(M_{q n}-m_{q n}\right) \beta}{\bar{\sigma}} T U_{\alpha}\left(q_{q n}, M_{q n}-1 ; n\right)+\frac{\frac{j \gamma}{M q n-m q n}}{\bar{\sigma}} T U_{\alpha}\left(q_{q n}, M_{q n} ; n\right)+\left(1-\frac{\alpha+\sigma+q_{q n} \zeta+M_{q n} \beta+\frac{j \gamma}{M q n-m q n}}{\bar{\sigma}}\right) * \\
& {\left[T U_{\alpha}\left(q_{q n}, m_{q n} ; n\right)-T U_{\alpha}\left(q_{q n}, M_{q n} ; n\right)\right]+\frac{\left(q_{q n}-m_{q n}\right) \zeta}{\bar{\sigma}} \operatorname{TU}_{\alpha}\left(q_{q n}, m_{q n} ; n\right)+\frac{\left(M_{q n}-m_{q n}\right) \beta}{\bar{\sigma}} \operatorname{TU}_{\alpha}\left(q_{q n}, m_{q n} ; n\right)-} \\
& \frac{\frac{i v}{M q n-m_{q n}}}{\bar{\sigma}} T U_{\alpha}\left(q_{q n}, M_{q n} ; n\right) \\
& =\frac{\frac{i \gamma}{M_{q n}-m_{q n}}}{\bar{\sigma}}\left[F+\left(M_{q n}-m_{q n}\right) r_{c}\right]+\frac{\sigma}{\bar{\sigma}}\left[T U_{\alpha}\left(q_{q n}+1, m_{q n} ; n\right)-T U_{\alpha}\left(q_{q n}+1, M_{q n} ; n\right)\right]+\frac{m_{q n} \zeta}{\bar{\sigma}} * \\
& {\left[T U_{\alpha}\left(q_{q n}-1, m_{q n} ; n\right)-T U_{\alpha}\left(q_{q n}-1, M_{q n} ; n\right)\right]+\frac{\left(q_{q n}-m_{q n}\right) \zeta}{\bar{\sigma}}\left[T U_{\alpha}\left(q_{q n}, m_{q n} ; n\right)-T U_{\alpha}\left(q_{q n}-1, M_{q n} ; n\right)\right]+} \\
& \frac{m_{q n} \beta}{\bar{\sigma}}\left[T U_{\alpha}\left(q_{q n}, m_{q n}-1 ; n\right)-T U_{\alpha}\left(q_{q n}, M_{q n}-1 ; n\right)\right]+\frac{\left(M_{q n}-m_{q n}\right) \beta}{\bar{\sigma}}\left[T U_{\alpha}\left(q_{q n}, m_{q n} ; n\right)-T U_{\alpha}\left(q_{q n}, M_{q n}-1 ; n\right)\right]+ \\
& \left(1-\frac{\alpha+\sigma+q_{q n} \zeta+M_{q n} \beta+\frac{j \gamma}{M_{q n}-m_{q n}}}{\bar{\sigma}}\right)\left[T U_{\alpha}\left(q_{q n}, m_{q n} ; n\right)-T U_{\alpha}\left(q_{q n}, M_{q n} ; n\right)\right] \\
& =\frac{\frac{j \gamma}{M q n-m_{q n}}}{\bar{\sigma}}\left[F+\left(M_{q n}-m_{q n}\right) r_{c}\right]+\frac{\sigma}{\bar{\sigma}}\left[T U_{\alpha}\left(q_{q n}+1, m_{q n} ; n\right)-T U_{\alpha}\left(q_{q n}+1, M_{q n} ; n\right)\right]+\frac{m_{q n} \zeta}{\bar{\sigma}} * \\
& {\left[T U_{\alpha}\left(q_{q n}-1, m_{q n} ; n\right)-U_{\alpha}\left(q_{q n}-1, M_{q n} ; n\right)\right]+\frac{\left(q_{q n}-m_{q n}\right) \zeta}{\bar{\sigma}}\left[T U_{\alpha}\left(q_{q n}, m_{q n} ; n\right)-T U_{\alpha}\left(q_{q n}, M_{q n} ; n\right)\right]+} \\
& \frac{m_{q n} \beta}{\bar{\sigma}}\left[T U_{\alpha}\left(q_{q n}, m_{q n}-1 ; n\right)-T U_{\alpha}\left(q_{q n}, M_{q n} ; n\right)\right]+\frac{\left(M_{q n}-m_{q n}\right) \beta}{\bar{\sigma}}\left[T U_{\alpha}\left(q_{q n}, m_{q n} ; n\right)-T U_{\alpha}\left(q_{q n}, M_{q n} ; n\right)\right]+ \\
& \left\{\left(1-\frac{\alpha+\sigma+q_{q n} \zeta+M_{q n} \beta+\frac{j \gamma}{M q n-m q n}}{\bar{\lambda}}\right)\left[T U_{\alpha}\left(q_{q n}, m_{q n} ; n\right)-T U_{\alpha}\left(q_{q n}, M_{q n} ; n\right)\right]-\frac{\left(q_{q n}-m_{q n}\right) \zeta}{\bar{\sigma}} *\right. \\
& \left.\left[T U_{\alpha}\left(q_{q n}-1, M_{q n} ; n\right)-T U_{\alpha}\left(q_{q n}, M_{q n} ; n\right)\right]-\frac{M_{q n} \beta}{\bar{\sigma}}\left[T U_{\alpha}\left(q_{q n}, M_{q n}-1 ; n\right)-T U_{\alpha}\left(q_{q n}, M_{q n} ; n\right)\right]\right\} .
\end{aligned}
$$

According to the induction hypothesis, the first six terms in the above (A3) are clearly increasing in $q_{q n}$.

To complete this proof, it need to be showed that $\Delta^{2}\left(q_{q n}, m_{q n} ; n+1\right)$ is also increasing in $q_{q n}$, where

$$
\begin{aligned}
& \Delta^{2}\left(q_{q n}, m_{q n} ; n+1\right)=\left(1-\frac{\alpha+\sigma+q_{q n} \zeta+M_{q n} \beta+\frac{j \gamma}{M_{q n}-m_{q n}}}{\bar{\sigma}}\right)\left[T U_{\alpha}\left(q_{q n}, m_{q n} ; n\right)-T U_{\alpha}\left(q_{q n}, M_{q n} ; n\right)\right] \\
& -\frac{\left(q_{q n}-m_{q n}\right) \zeta}{\bar{\sigma}}\left[T U_{\alpha}\left(q_{q n}-1, M_{q n} ; n\right)-T U_{\alpha}\left(q_{q n}, M_{q n} ; n\right)\right]-\frac{M_{q n} \beta}{\bar{\sigma}}\left[T U_{\alpha}\left(q_{q n}, M_{q n}-1 ; n\right)-T U_{\alpha}\left(q_{q n}, M_{q n} ; n\right)\right] .
\end{aligned}
$$


Here we use the same assumption $\bar{\sigma} \geq \alpha+\sigma+M_{q n}^{*} \beta+M_{q n}^{*} \zeta+j \gamma$ as case 1 . Then we rearrange $\Delta^{2}\left(q_{q n}, m_{q n} ; n+1\right)$ as follows:

$$
\begin{aligned}
& \Delta^{2}\left(q_{q n}, m_{q n} ; n+1\right)=\left(1-\frac{\alpha+\sigma+M_{q n}^{*} \zeta+M_{q n}^{*} \beta+\frac{j \gamma}{M_{q n}-m_{q n}}}{\bar{\sigma}}\right)\left[T U_{\alpha}\left(q_{q n}, m_{q n} ; n\right)-T U_{\alpha}\left(q_{q n}, M_{q n} ; n\right)\right] \\
& +\left(1-\frac{\left.\left(M_{q n}^{*}-q_{q n}\right) \zeta+\left(M_{q n}^{*}-M_{q n}\right) \beta+\frac{j \gamma}{M_{q n}-m_{q n}}\right)}{\bar{\sigma}}\right)\left[T U_{\alpha}\left(q_{q n}, m_{q n} ; n\right)-T U_{\alpha}\left(q_{q n}, M_{q n} ; n\right)\right] \\
& -\frac{\left(q_{q n}-m_{q n}\right) \zeta}{\bar{\sigma}}\left[T U_{\alpha}\left(q_{q n}-1, M_{q n} ; n\right)-T U_{\alpha}\left(q_{q n}, M_{q n} ; n\right)\right]-\frac{M_{q n} \beta}{\bar{\sigma}}\left[T U_{\alpha}\left(q_{q n}, M_{q n}-1 ; n\right)-T U_{\alpha}\left(q_{q n}, M_{q n} ; n\right)\right] .
\end{aligned}
$$

In the above expression, according to the induction hypothesis, the first two items are clearly increasing in $q_{q n}$. Due to large $M_{q n}^{*}$, the coefficient of the second increasing item $\frac{\left(M_{q n}^{*}-q_{q n}\right) \zeta+\left(M_{q n}^{*}-M_{q n}\right) \beta}{\bar{\sigma}}$ is almost near 1 , as the coefficients of the last two decreasing items are extremely tiny. According to the outcome of Lemma 2, it is obvious that $T U_{\alpha}\left(q_{q n}, m_{q n} ; n\right)-T U_{\alpha}\left(q_{q n}+1, m_{q n} ; n\right)$ is bounded for every state $\left(q_{q n}, m_{q n}\right)$. Therefore, the entire expression of $\Delta^{2}\left(q_{q n}, m_{q n} ; n+1\right)$ should be increasing in $q_{q n}$ for $M_{q n}^{*}$ sufficiently large.

Therefore, $T U_{\alpha}\left(q_{q n}, m_{q n} ; n+1\right)-T U_{\alpha}\left(q_{q n}, M_{q n} ; n+1\right)$ is increasing in $q_{q n}$ when $m_{q n} \leq q_{q n}<M_{q n}$.

Case 3. $q_{q n}<m_{q n}$

In this case, $\min \left(q_{q n}, M_{q n}\right)=\min \left(q_{q n}, m_{q n}\right)=q_{q n}$, therefore (A2) becomes

$$
\begin{aligned}
& T U_{\alpha}\left(q_{q n}, m_{q n} ; n+1\right)-T U_{\alpha}\left(q_{q n}, M_{q n} ; n+1\right) \\
& =\frac{\frac{j \gamma}{M_{q n}-m_{q n}}}{\bar{\sigma}}\left[F+\left(M_{q n}-m_{q n}\right) r_{c}\right]+\frac{\sigma}{\bar{\sigma}}\left[T U_{\alpha}\left(q_{q n}+1, m_{q n} ; n\right)-T U_{\alpha}\left(q_{q n}+1, M_{q n} ; n\right)\right]+ \\
& \frac{q_{q n} \zeta}{\bar{\sigma}}\left[T U_{\alpha}\left(q_{q n}-1, m_{q n} ; n\right)-T U_{\alpha}\left(q_{q n}-1, M_{q n} ; n\right)\right]+\frac{m_{q n} \beta}{\bar{\sigma}}\left[T U_{\alpha}\left(q_{q n}, m_{q n}-1 ; n\right)-T U_{\alpha}\left(q_{q n}, M_{q n}-1 ; n\right)\right] \\
& -\frac{\left(M_{q n}-m_{q n}\right) \beta}{\bar{\sigma}} T U_{\alpha}\left(q_{q n}, M_{q n}-1 ; n\right)+\frac{\frac{j \gamma}{M_{q n}-m_{q n}}}{\bar{\sigma}} T U_{\alpha}\left(q_{q n}, M_{q n} ; n\right)+\left(1-\frac{\alpha+\sigma+q_{q n} \zeta+M_{q n} \beta+\frac{j \gamma}{M_{q n}-m_{q n}}}{\bar{\sigma}}\right) * \\
& {\left[T U_{\alpha}\left(q_{q n}, m_{q n} ; n\right)-T U_{\alpha}\left(q_{q n}, M_{q n} ; n\right)\right]+\frac{\left(M_{q n}-m_{q n}\right) \beta}{\bar{\sigma}} T U_{\alpha}\left(q_{q n}, m_{q n} ; n\right)-\frac{\frac{j \gamma}{M_{q n} n} \overline{m_{q n}}}{\bar{\sigma}} T U_{\alpha}\left(q_{q n}, M_{q n} ; n\right)} \\
& =\frac{\frac{j p}{M q n-m_{q n}}}{\bar{\sigma}}\left[F+\left(M_{q n}-m_{q n}\right) r_{c}\right]+\frac{\sigma}{\bar{\sigma}}\left[T U_{\alpha}\left(q_{q n}+1, m_{q n} ; n\right)-T U_{\alpha}\left(q_{q n}+1, M_{q n} ; n\right)\right] \\
& +\frac{q_{q n} \zeta}{\bar{\sigma}}\left[T U_{\alpha}\left(q_{q n}-1, m_{q n} ; n\right)-T U_{\alpha}\left(q_{q n}-1, M_{q n} ; n\right)\right]+\frac{m_{q n} \beta}{\bar{\sigma}}\left[T U_{\alpha}\left(q_{q n}, m_{q n}-1 ; n\right)-T U_{\alpha}\left(q_{q n}, M_{q n}-1 ; n\right)\right] \\
& +\frac{\left(M_{q n}-m_{q n}\right) \beta}{\bar{\sigma}}\left[T U_{\alpha}\left(q_{q n}, m_{q n} ; n\right)-T U_{\alpha}\left(q_{q n}, M_{q n}-1 ; n\right)\right]+\left(1-\frac{\alpha+\sigma+q_{q n} \zeta+M_{q n} \beta+\frac{j \gamma}{M q n-m q n}}{\bar{\lambda}}\right) * \\
& {\left[T U_{\alpha}\left(q_{q n}, m_{q n} ; n\right)-T U_{\alpha}\left(q_{q n}, M_{q n} ; n\right)\right]} \\
& =\frac{\frac{i \gamma}{M q \eta n}-m_{q n}}{\bar{\sigma}}\left[F+\left(M_{q n}-m_{q n}\right) r_{c}\right]+\frac{\sigma}{\bar{\sigma}}\left[T U_{\alpha}\left(q_{q n}+1, m_{q n} ; n\right)-T U_{\alpha}\left(q_{q n}+1, M_{q n} ; n\right)\right] \\
& +\frac{q_{q n} \zeta}{\bar{\sigma}}\left[T U_{\alpha}\left(q_{q n}-1, m_{q n} ; n\right)-T U_{\alpha}\left(q_{q n}-1, M_{q n} ; n\right)\right]+\frac{m_{q n} \beta}{\bar{\sigma}}\left[T U_{\alpha}\left(q_{q n}, m_{q n}-1 ; n\right)-T U_{\alpha}\left(q_{q n}, M_{q n} ; n\right)\right] \\
& +\frac{\left(M_{q n}-m_{q n}\right) \beta}{\bar{\sigma}}\left[T U_{\alpha}\left(q_{q n}, m_{q n} ; n\right)-T U_{\alpha}\left(q_{q n}, M_{q n} ; n\right)\right]+\left\{\left(1-\frac{\alpha+\sigma+q_{q n} \zeta+M_{q n} \beta+\frac{j \gamma}{M q n n}-m_{q n}}{\bar{\lambda}}\right) *\right. \\
& \left.\left[T U_{\alpha}\left(q_{q n}, m_{q n} ; n\right)-T U_{\alpha}\left(q_{q n}, M_{q n} ; n\right)\right]-\frac{M_{q n} \beta}{\bar{\sigma}}\left[T U_{\alpha}\left(q_{q n}, M_{q n}-1 ; n\right)-T U_{\alpha}\left(q_{q n}, M_{q n} ; n\right)\right]\right\} .
\end{aligned}
$$

According to the induction hypothesis, the first five items in above (A4) are increasing in $q_{q n}$. To complete the proof, it needs to be showed that $\Delta^{3}\left(q_{q n}, m_{q n} ; n+1\right)$ is increasing in $q_{q n}$, where

$$
\begin{aligned}
& \Delta^{3}\left(q_{q n}, m_{q n} ; n+1\right)=\left(1-\frac{\alpha+\sigma+q_{q n} \zeta+M_{q n} \beta+\frac{j \gamma}{M q n-m q n}}{\bar{\sigma}}\right)\left[T U_{\alpha}\left(q_{q n}, m_{q n} ; n\right)-T U_{\alpha}\left(q_{q n}, M_{q n} ; n\right)\right] \\
& -\frac{M_{q n} \beta}{\bar{\sigma}}\left[T U_{\alpha}\left(q_{q n}, M_{q n}-1 ; n\right)-T U_{\alpha}\left(q_{q n}, M_{q n} ; n\right)\right] .
\end{aligned}
$$


We first use the same assumption $\bar{\sigma} \geq \alpha+\sigma+M_{q n}^{*} \beta+q_{q n} \zeta+j \gamma$ as case 1 and case 2 , then we rearrange $\Delta^{3}\left(q_{q n}, m_{q n} ; n+1\right)$ as follows:

$$
\begin{aligned}
& \Delta^{3}\left(q_{q n}, m_{q n} ; n+1\right) \\
& =\left(1-\frac{\alpha+\sigma+q_{q n} \zeta+M_{q n}^{*} \beta+\frac{j \gamma}{M_{q n}-m_{q n}}}{\bar{\lambda}}\right)\left[T U_{\alpha}\left(q_{q n}, m_{q n} ; n\right)-T U_{\alpha}\left(q_{q n}, M_{q n} ; n\right)\right]+\frac{\left(M_{q n}^{*}-M_{q n}\right) \beta}{\bar{\sigma}} * \\
& {\left[T U_{\alpha}\left(q_{q n}, m_{q n} ; n\right)-T U_{\alpha}\left(q_{q n}, M_{q n} ; n\right)\right]-\frac{M_{q n} \beta}{\bar{\sigma}}\left[T U_{\alpha}\left(q_{q n}, M_{q n}-1 ; n\right)-T U_{\alpha}\left(q_{q n}, M_{q n} ; n\right)\right] .}
\end{aligned}
$$

In the above expression, by the induction hypothesis, the first two terms are clearly increasing in $q_{q n}$. Due to extremely largest $M_{q n}^{*}$, the coefficient of the second item, $\frac{\left(M_{q n}^{*}-M_{q n}\right) \beta}{\bar{\sigma}}$ is almost near 1, while the coefficients of the last item is extremely small. According to the outcome of Lemma 2, it is obvious that $T U_{\alpha}\left(q_{q n}, m_{q n} ; n\right)-T U_{\alpha}\left(q_{q n}+1, m_{q n} ; n\right)$ is bounded for every state $\left(q_{q n}, m_{q n}\right)$. Therefore, according to a similar argument in case 1 and case 2 , the entire expression of $\Delta^{3}\left(q_{q n}, m_{q n} ; n+1\right)$ should be increasing in $q_{q n}$ for sufficiently largest $M_{q n}^{*}$.

Depending on the outcomes of the above three cases, it can be concluded that $T U_{\alpha}\left(q_{q n}, m_{q n} ; n+1\right)-$ $T U_{\alpha}\left(q_{q n}, M_{q n} ; n+1\right)$ is increasing in $q_{q n}$ when $T U_{\alpha}\left(q_{q n}, m_{q n} ; n+1\right)=T U_{\alpha}^{2}\left(q_{q n}, m_{q n} ; n+1\right)$.

(2) $T U_{\alpha}\left(q_{q n}, m_{q n}\right)=T U_{\alpha}^{1}\left(q_{q n}, m_{q n}\right)$ :

This proof will be implemented by induction on $n$. From (9), for $n=1$, it is noted that

$$
T U_{\alpha}\left(q_{q n}, m_{q n} ; n=1\right)-T U_{\alpha}\left(q_{q n}, M_{q n} ; n=1\right)=\frac{k q_{q n}}{\bar{\sigma}}-\frac{k q_{q n}}{\bar{\sigma}}=0
$$

It is clear that $T U_{\alpha}\left(q_{q n}, m_{q n} ; n=1\right)-T U_{\alpha}\left(q_{q n}, M_{q n} ; n=1\right)$ is non-decreasing in $q_{q n}$.

From the above result, it can be assumed that $T U_{\alpha}\left(q_{q n}, m_{q n} ; n\right)-T U_{\alpha}\left(q_{q n}, M_{q n} ; n\right)$ is increasing in $q_{q n}$, then it need to be proved that $T U_{\alpha}\left(q_{q n}, m_{q n} ; n+1\right)-T U_{\alpha}\left(q_{q n}, M_{q n} ; n+1\right)$ is also increasing in $q_{q n}$. From (9), we have

$$
\begin{aligned}
& T U_{\alpha}\left(q_{q n}, m_{q n} ; n+1\right)-T U_{\alpha}\left(q_{q n}, M_{q n} ; n+1\right) \\
& =\frac{\sigma}{\bar{\sigma}}\left[T U_{\alpha}\left(q_{q n}+1, m_{q n} ; n\right)-T U_{\alpha}\left(q_{q n}+1, M_{q n} ; n\right)\right]+\left[\frac{\zeta \min \left(q_{q n}, m_{q n}\right)}{\bar{\sigma}} T U_{\alpha}\left(q_{q n}-1, m_{q n} ; n\right)\right. \\
& \left.-\frac{\zeta \min \left(q_{q n}, M_{q n}\right)}{\bar{\sigma}} T U_{\alpha}\left(q_{q n}-1, M_{q n} ; n\right)\right]+\left[\frac{m_{q n} \beta}{\bar{\sigma}} T U_{\alpha}\left(q_{q n}, m_{q n}-1 ; n\right)-\frac{M_{q n} \beta}{\bar{\sigma}} T U_{\alpha}\left(q_{q n}, M_{q n}-1 ; n\right)\right] \\
& +\left[\left(1-\frac{\alpha+\sigma\left(q_{q n}, m_{q n}\right)}{\bar{\sigma}}\right) T U_{\alpha}\left(q_{q n}, m_{q n} ; n\right)-\left(1-\frac{\alpha+\sigma\left(q_{q n}, M_{q n}\right)}{\bar{\sigma}}\right) T U_{\alpha}\left(q_{q n}, M_{q n} ; n\right)\right] \\
& =\frac{\sigma}{\bar{\sigma}}\left[T U_{\alpha}\left(q_{q n}+1, m_{q n} ; n\right)-T U_{\alpha}\left(q_{q n}+1, M_{q n} ; n\right)\right]+\left[\frac{\zeta \min \left(q_{q n}, m_{q n}\right)}{\bar{\sigma}} T U_{\alpha}\left(q_{q n}-1, m_{q n} ; n\right)\right. \\
& \left.-\frac{\zeta \min \left(q_{q n}, M_{q n}\right)}{\bar{\sigma}} T U_{\alpha}\left(q_{q n}-1, M_{q n} ; n\right)\right]+\left[\frac{m \beta}{\bar{\sigma}} T U_{\alpha}\left(q_{q n}, m_{q n}-1 ; n\right)-\frac{M_{q n} \beta}{\bar{\sigma}} T U_{\alpha}\left(q_{q n}, M_{q n}-1 ; n\right)\right] \\
& +\frac{\frac{j \gamma}{M_{q n}-m_{q n}}}{\bar{\sigma}} T U_{\alpha}\left(q_{q n}, M_{q n} ; n\right)+\left[\left(1-\frac{\left.\alpha+\sigma\left(q_{q n}, m_{q n}\right)+\frac{j \gamma}{M_{q n}-m_{q n}}\right) T U_{\alpha}\left(q_{q n}, m_{q n} ; n\right)}{\bar{\sigma}}\right.\right. \\
& \left.-\left(1-\frac{\alpha+\sigma\left(q_{q n}, M_{q n}\right)}{\bar{\sigma}}\right) T U_{\alpha}\left(q_{q n}, M_{q n} ; n\right)\right]+\frac{\frac{j \gamma}{M_{q n}-m_{q n}}}{\bar{\sigma}} T U_{\alpha}\left(q_{q n}, m_{q n} ; n\right)-\frac{\overline{j \gamma}}{\overline{M_{q n}-m_{q n}}} T U_{\alpha}\left(q_{q n}, M_{q n} ; n\right) .
\end{aligned}
$$

Clearly, the Equation (A5) is the above Equation (A1) plus the last two items. It is obvious that the part of last two items is increasing to $q_{q n}$. Moreover, according to the previous result of Equation (A1), it can be concluded that $T U_{\alpha}\left(q_{q n}, m_{q n} ; n+1\right)-T U_{\alpha}\left(q_{q n}, M_{q n} ; n+1\right)$ is increasing in $q$ when $T U_{\alpha}\left(q_{q n}, m_{q n} ; n+1\right)=T U_{\alpha}^{1}\left(q_{q n}, m_{q n} ; n+1\right)$.

Since we have first proved $T U_{\alpha}\left(q_{q n}, m_{q n} ; n+1\right)-T U_{\alpha}\left(q_{q n}, M_{q n} ; n+1\right)$ is increasing in $q_{q n}$ when $T U_{\alpha}\left(q_{q n}, m_{q n} ; n+1\right)=T U_{\alpha}^{2}\left(q_{q n}, m_{q n} ; n+1\right)$, it can be concluded that $T U_{\alpha}\left(q_{q n}, m_{q n} ; n+1\right)-$ $T U_{\alpha}\left(q_{q n}, M_{q n} ; n+1\right)$ is increasing in $q_{q n}$.

Based on standard arguments applying successive approximation for discounted MDPs, $T U_{\alpha}\left(q_{q n}, m_{q n}\right)-T U_{\alpha}\left(q_{q n}, M_{q n}\right)=\lim _{n}\left\{T U_{\alpha}\left(q_{q n}, m_{q n} ; n\right)-T U_{\alpha}\left(q_{q n}, M_{q n} ; n\right)\right\}$ is certainly increasing in $q_{q n}$. Consequently, the proof is done. 


\section{References}

1. Sheu, S.H.; Chien, Y.H. Optimal Age-replacement Policy of System Subject to Shocks with Random Lead-time. Eur. J. Oper. Res. 2004, 159, 132-144. [CrossRef]

2. Scarf, P.A.; Dwight, R.; Al-Musrati, A. On Reliability Criterion and the Implied Cost of Failure for a Maintained Component. Reliab. Eng. Syst. Saf. 2005, 89, 199-207. [CrossRef]

3. Chien, Y.H. Optimal age for preventive replacement under a combined fully renewable free replacement with a pro-rata warranty. Int. J. Prod. Econ. 2010, 124, 198-205. [CrossRef]

4. Lim, J.H.; Qu, J.; Zuo, M.J. Age replacement policy based on imperfect repair with random probability. Reliab. Eng. Syst. Saf. 2016, 149, 24-33. [CrossRef]

5. Park, M.; Pham, H. Cost models for age replacement policies and block replacement policies under warranty. Appl. Math. Model. 2016, 40, 5689-5702. [CrossRef]

6. Jin, L.; Yamamoto, W. Adaptive Age Replacement Using On-Line Monitoring. Procedia Eng. 2017, 174, 117-125. [CrossRef]

7. Zhao, X.; Al-Khalifa, K.N.; Hamouda, A.M.; Nakagawa, T. Age replacement models: A summary with new perspectives and methods. Reliab. Eng. Syst. Saf. 2017, 161, 95-105. [CrossRef]

8. Scarf, P.A. On the application of mathematical models in maintenance. Eur. J. Oper. Res. 1997, 99, 493-506. [CrossRef]

9. Wang, H. A survey of maintenance policies of deteriorating systems. Eur. J. Oper. Res. 2002, 139, 469-489. [CrossRef]

10. Kyriakidis, E.G.; Dimitrakos, T.D. Optimal preventive maintenance of a production system with an intermediate buffer. Eur. J. Oper. Res. 2006, 168, 86-99. [CrossRef]

11. Dimitrakos, T.D.; Kyriakidis, E.G. A semi-Markov decision algorithm for the maintenance of a production system with buffer capacity and continuous repair times. Int. J. Prod. Econ. 2008, 111, 752-762. [CrossRef]

12. Berthaut, F.; Gharbi, A.; Kenne, J.-P.; Boulet, J.-F. Improved joint preventive maintenance and hedging point policy. Int. J. Prod. Econ. 2010, 127, 60-72. [CrossRef]

13. Wu, J.; Xie, M.; Ng, T.S.A. On a general periodic preventive maintenance policy incorporating warranty contracts and system ageing losses. Int. J. Prod. Econ. 2011, 129, 102-111. [CrossRef]

14. Huang, Y.S.; Huang, C.D.; Ho, J.W. A customized two-dimensional extended warranty with preventive maintenance. Eur. J. Oper. Res. 2017, 257, 971-978. [CrossRef]

15. Shafiee, M.; Finkelstein, M. An optimal age-based group maintenance policy for multi-unit degrading systems. Reliab. Eng. Syst. Saf. 2015, 134, 230-238. [CrossRef]

16. Babishin, V.; Taghipour, S. A multicomponent system subject to hard and hidden soft failures is considered. Appl. Math. Model. 2016, 40, 10480-10505. [CrossRef]

17. Li, H.; Xu, S.H. On the coordinated Random Group Replacement Policy in Multivariate Repairable Syst. Oper. Res. 2004, 52, 464-477. [CrossRef]

18. Olde Keizer, M.C.A.; Flapper, S.D.P.; Teunte, R.H. Condition-based maintenance policies for systems with multiple dependent components: A review. Eur. J. Oper. Res. 2017, 261, 405-420. [CrossRef]

19. Alaswad, S.; Xiang, Y. A review on condition-based maintenance optimization models for stochastically deteriorating system. Reliab. Eng. Syst. Saf. 2017, 157, 54-63. [CrossRef]

20. Neuts, M.F.; Lucantoni, D.M. A Markovian queue with N servers subject to breakdowns and repairs. Manag. Sci. 1979, 25, 849-861. [CrossRef]

21. Neuts, M.F. Matrix-Geometric Solutions in Stochastic Models-An Algorithmic Approach; Dover: New York, NY, USA, 1981; pp. 1-14.

22. Liu, G.S. Three $\mathrm{m}$-failure group maintenance models for $\mathrm{M} / \mathrm{M} / \mathrm{N}$ unreliable queuing service systems. Comput. Ind. Eng. 2012, 62, 1011-1024. [CrossRef]

23. Liu, G.S. Dynamic group instantaneous replacement policies for unreliable Markovian Service Systems. Int. J. Prod. Econ. 2011, 130, 203-217. [CrossRef]

24. Aizpurua, J.I.; Catterson, V.M.; Papadopoulos, Y.; Chiacchio, F.; D’Urso, D. Supporting group maintenance through prognostics-enhanced dynamic dependability prediction. Reliab. Eng. Syst. Saf. 2017, 168, 171-188. [CrossRef]

25. Li, D.; Ren, M.; Meng, G. Application of Internet of Things Technology on Predictive Maintenance System of Coal Equipment. Procedia Eng. 2017, 174, 885-889. 
26. Hernandez-Lerma, O.; Lassere, J.B. Discrete-Time Markov Control Processes, 1st ed.; Springer: New York, NY, USA, 1996.

27. Puterman, M.L. Markov Decision Processes-Discrete Stochastic Dynamic Programming; Wiley: New York, NY, USA, 1994; pp. 560-573.

28. Tijms, H.C. Stochastic Models-An Algorithmic Approach; Wiley: New York, NY, USA, 1994; pp. 154-156.

(C) (1)

(C) 2019 by the author. Licensee MDPI, Basel, Switzerland. This article is an open access article distributed under the terms and conditions of the Creative Commons Attribution (CC BY) license (http://creativecommons.org/licenses/by/4.0/). 